\title{
STABILITY OF SHEAR BANDS IN AN ELASTOPLASTIC MODEL FOR GRANULAR FLOW: THE ROLE OF DISCRETENESS
}

\author{
Michael Shearer*, David G. Schaeffer ${ }^{\dagger}$ and Thomas P. Witelski ${ }^{\ddagger}$
}

\begin{abstract}
Continuum models for granular flow generally give rise to systems of nonlinear partial differential equations that are linearly ill-posed. In this paper we introduce discreteness into an elastoplasticity model for granular flow by approximating spatial derivatives with finite differences. The resulting ordinary differential equations have bounded solutions for all time, a consequence of both discreteness and nonlinearity.

We study how the large-time behavior of solutions in this model depends on an elastic shear modulus $\mathcal{E}$. For large and moderate values of $\mathcal{E}$, the model has stable steady-state solutions with uniform shearing except for one shear band; almost all solutions tend to one of these as $t \rightarrow \infty$. However, when $\mathcal{E}$ becomes sufficiently small, the single-shear-band solutions lose stability through a Hopf bifurcation. The value of $\mathcal{E}$ at the bifurcation point is proportional to the ratio of the mesh size to the macroscopic length scale. These conclusions are established analytically through a careful estimation of the eigenvalues. In numerical simulations we find that: (i) after stability is lost, time-periodic solutions appear, containing both elastic and plastic waves, and (ii) the bifurcation diagram representing these solutions exhibits bi-stability.
\end{abstract}

\section{Introduction}

Continuum models for granular flow, based on elastoplasticity, generally give rise to ill-posed systems of nonlinear partial differential equations [1-5]. The consequence of ill-posedness of the linearized equations is that small-amplitude short-wave-length disturbances are amplified catastrophically. Although ill-posedness seems to be connected to the formation of small-scale structures such as shear bands, understanding this connection has been hampered by the difficulties of interpreting ill-posed PDEs.

In this paper and its predecessor [6], we attempt to these issues in the context of an antiplane-shear model [7]. Our results indicate that the discreteness of granular materials may play a crucial role. We explore the effects of discreteness by studying a caricature of nearest neighbor interaction forces given by a finite-difference approximation of the continuum equations (more accurately, a continuous-time/discrete-

* Department of Mathematics and Center for Research in Scientific Computation, North Carolina State University, Raleigh, NC 27695. Research support under NSF Grants DMS 9818900, DMS 0204578 is gratefully acknowledged.

${ }^{\dagger}$ Department of Mathematics and Center for Nonlinear and Complex Systems, Duke University, Durham, NC 27708-0320. Research support under NSF Grant DMS 9803305 is gratefully acknowledged.

$\ddagger$ Department of Mathematics and Center for Nonlinear and Complex Systems, Duke University, Durham, NC 27708-0320. Supported by a research fellowship from the Alfred P. Sloan foundation. 
space approximation). For both a granular medium and a finite-difference approximation, there is a length scale $\delta$ below which the continuum theory becomes inappropriate - the grain size or the mesh parameter, respectively. Even though a constitutive law for granular materials that accurately models effective contact forces and nearest neighbor interactions is not yet available, by considering the finite-difference approximation we obtain a discrete model that is suggestive of the microscopic physics while retaining the macroscopic physics given by the continuum equations. There is a vast literature on both continuum and discrete modeling of granular materials, see [8]. An indication of the breadth of approaches can be learned from the recent book of review articles [9], and the conference proceedings [10]. In particular, these references contain articles on micromechanical models of granular materials, and on the kinetic theory of rapid granular flow. These important recent developments represent approaches to the dynamics of granular flow rather different from that taken here.

In [6], the effects of elasticity were neglected. By contrast, in this paper, we focus on the influence of such effects. In both [6] and this paper, we consider time evolution in a one-dimensional problem extracted from the antiplane-shear model [7]. The discrete-space model of the continuum mechanics PDE problem is a system of $3 N$ ODEs of the form

$$
\begin{aligned}
\frac{d w_{n}}{d t} & =\frac{p_{n}-p_{n-1}}{\delta}, \\
\mathcal{E}^{-1} \frac{d}{d t}\left(\begin{array}{c}
p_{n} \\
q_{n}
\end{array}\right) & =\mathbf{f}\left(\frac{w_{n+1}-w_{n}}{\delta}, \frac{p_{n}-p_{n-1}}{\delta}, \frac{q_{n}-q_{n-1}}{\delta}, p_{n}, q_{n}\right),
\end{aligned}
$$

for $n=1,2, \cdots N$, where $w_{n}$ describes the local velocity, $p_{n}, q_{n}$ are components of the local stress tensor and $\mathbf{f} \in \mathbb{R}^{2}$ is a nonlinear function that combines constitutive information for both elastic and plastic deformations. The mesh parameter $\delta=1 / N$, and $\mathcal{E}$ is a dimensionless constant.

This problem admits a homogeneous steady-state solution in which material undergoes uniform shear (see Figure 1(left)). However, under certain conditions, this homogeneous state is unstable; in fact, the corresponding continuum equations are linearly ill-posed with respect to small perturbations about the uniform shear solution. The ODE system (1.1) mimics the ill-posedness of the PDEs in that the linearization of the ODEs at the homogeneous state has numerous positive eigenvalues, ranging up to a maximum eigenvalue that is $O(1 / \delta)$ as $\delta \rightarrow 0$. Moreover, the eigenvalues of the discrete problem converge to the eigenvalues of the continuous problem as $\delta \rightarrow 0$, the latter set being unbounded. Besides the homogeneous steady state, there are many other steady-state solutions of this problem. In this paper we study the stability of solutions with a single shear band and uniform shearing elsewhere (see Figure 1(right)).

As discussed in Section 2.2 below, the importance of elasticity effects may be quantified in terms of the dimensionless parameter $\mathcal{E}$ that is proportional to $G U^{2}$, where $G$ is the elastic shear modulus and $U$ is a characteristic velocity. The problem 
in [6] corresponds to the limit $\mathcal{E} \rightarrow \infty$; however, as shown in [7], the regime of small $\mathcal{E}$ is more physical for granular-flow applications. In the present paper, we establish two conclusions analytically:

- For any small $\delta$, there is a critical value for $\mathcal{E}$ such that if $\mathcal{E}>\mathcal{E}_{\text {crit }}(\delta)$, the single-shear-band steady solutions are asymptotically stable. As $\delta$ tends to zero, $\mathcal{E}_{\text {crit }}(\delta)=E_{\text {crit }} \delta$ to leading order, and we calculate the positive constant $E_{\text {crit }}$.

- If $\mathcal{E}<\mathcal{E}_{\text {crit }}(\delta)$, the single-shear-band solutions are unstable. As $\mathcal{E}$ crosses $\mathcal{E}_{\text {crit }}(\delta)$ while $\delta$ is held fixed, two complex-conjugate eigenvalues cross the imaginary axis: i.e., a Hopf bifurcation occurs.

We also make two observations based on a limited number of numerical simulations:

- When the single-shear-band solutions are stable, transients in the dynamical problem exhibit the scaling behavior seen in [6], see Section 5.5.

- For $\mathcal{E}<\mathcal{E}_{\text {crit }}(\delta)$, starting from a small perturbation of the single-shear-band equilibrium, solutions of the dynamical problem evolve to time-periodic behavior as $t \rightarrow \infty$. The strength of the shear band varies periodically. Elastic waves and elastic-plastic interfaces [11] propagate through the sample, and changes in the strength of the shear band are triggered by their arrival. Interestingly, the amplitude of the periodic solutions does not decay to zero as the critical value is approached; instead, there is a hysteresis, and it seems likely that this is linked to the Hopf bifurcation through an unstable branch of periodic solutions.

The remainder of this paper is organized as follows. In Section 2 we formulate the differential equations to be solved, starting from a system of PDEs in two space dimensions and time and reducing it to a system of ODEs (in time) that approximates the PDEs in one space variable. In Section 3 we characterize the single-shear-band equilibria of the ODEs and formulate an eigenvalue problem for their stability. In Section 4, we use a perturbation theory approach to estimate the linear stability condition. In Section 5, we carry out a direct calculation of the full spectrum of eigenvalues to rigorously prove the stability result. Finally, numerical results are presented in Section 6.

\section{The Antiplane-Shear Model}

In this section, we derive the discrete model (1.1) that is the focus of this paper. In the first three subsections, we state the continuum antiplane shear model (Section 2.1), discuss its nondimensionalization (Section 2.2), and formulate the problem for one-dimensional perturbations of uniform shear (Section 2.3), leading 

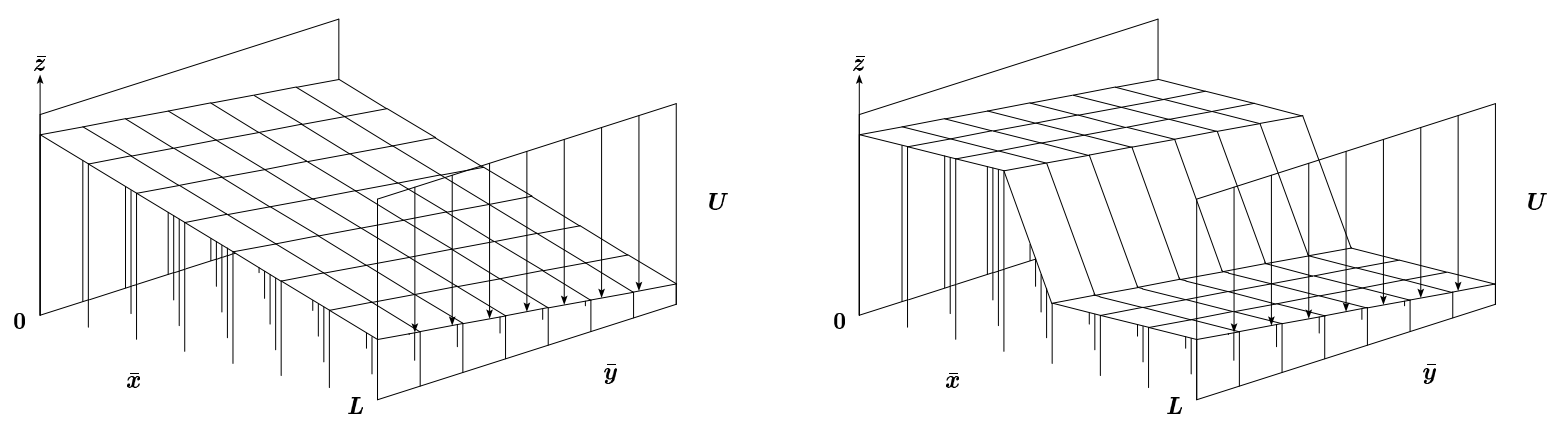

Figure 1: The uniform solution (left) and a solution with one shear band (right)

to the one-dimensional continuum equations, whose spatial discretization leads immediately to the discrete model. In Subsection 2.3, we also describe ill-posedness of the continuum equations.

\subsection{Continuum equations in two space dimensions}

The equations for two-dimensional flow of Coulomb materials share key mathematical properties, in particular ill-posedness, with the anti-plane shear model that we consider (cf [5]). In this model, all material points move (in three dimensions) in straight lines parallel to a given direction, say the $\bar{z}$-axis ${ }^{\S}$. The unknowns have the reduced structure:

$$
\text { velocity: }(0,0, \bar{v}) \quad \text { stress: }\left(\begin{array}{ccc}
\bar{c} & 0 & \bar{p} \\
0 & \bar{c} & \bar{q} \\
\bar{p} & \bar{q} & \bar{c}
\end{array}\right) .
$$

The velocity and the two off-diagonal stress components, $\bar{p}$ and $\bar{q}$, are functions of $\bar{x}, \bar{y}$ and $\bar{t}$ but not of $\bar{z}$. The diagonal component of the stress tensor, $\bar{c}$, is a constant, independent of all the coordinates and time. Mass is conserved because the flow is incompressible, but energy is dissipated by friction.

Let $\overline{\boldsymbol{\tau}}=(\bar{p}, \bar{q})^{\top}$ be the column vector of non-constant components of the stress. In elastoplasticity, the stress is constrained to lie inside the yield surface, here given by the inequality

$$
|\overline{\boldsymbol{\tau}}| \leq Y,
$$

in which $Y$ is the yield stress. While $Y$ is related to the confining pressure $\bar{c}$, we treat $Y$ as a given parameter and henceforth ignore $\bar{c}$.

The governing equations consist of conservation of momentum and a constitutive

\footnotetext{
$\S$ Overbars indicate variables with dimensions. These will be removed in the nondimensionalization below.
} 
law. In antiplane shear, the former reduces to a single equation

$$
\rho \frac{\partial \bar{v}}{\partial \bar{t}}=\nabla \cdot \bar{\tau} \equiv \frac{\partial \bar{p}}{\partial \bar{x}}+\frac{\partial \bar{q}}{\partial \bar{y}},
$$

where $\rho$, the density, is a constant. The constitutive equation, which relates the stress $\overline{\boldsymbol{\tau}}$ to the strain rate $\nabla \bar{v}$, has the form

$$
G^{-1} \frac{\partial \bar{\tau}}{\partial \bar{t}}=\nabla \bar{v}-\overline{\mathcal{P}}(\overline{\boldsymbol{\tau}}, \nabla \bar{v})
$$

where $G$ is the elastic shear modulus and $\overline{\mathcal{P}}$, the plastic strain rate, is a term that acts to prevent violations of the yield condition (2.1). As described in [7], this term is modeled by the formula

$$
\overline{\mathcal{P}}=\left\{\begin{array}{cl}
\frac{\overline{\boldsymbol{\tau}} \cdot \nabla \bar{v}}{Y^{2} \cos \alpha} \mathbf{R}_{\alpha}^{\top} \overline{\boldsymbol{\tau}} & \text { if }|\overline{\boldsymbol{\tau}}|=Y \text { and } \overline{\boldsymbol{\tau}} \cdot \nabla \bar{v}>0 \\
0 & \text { otherwise }
\end{array}\right.
$$

where $\alpha$ is a given angle satisfying $0<\alpha<\pi / 2, \mathbf{R}_{\alpha}$ denotes counter-clockwise rotation through an angle $\alpha$

$$
\mathbf{R}_{\alpha}=\left(\begin{array}{rr}
\cos \alpha & -\sin \alpha \\
\sin \alpha & \cos \alpha
\end{array}\right)
$$

and $\mathbf{R}_{\alpha}^{\top}$ denotes the transpose of this matrix. In (2.4), we shall refer to the equation $|\overline{\boldsymbol{\tau}}|=Y$ as the yield condition and to the inequality $\overline{\boldsymbol{\tau}} \cdot \nabla \bar{v}>0$ as the loading condition. This flow rule is nonassociative: i.e., the plastic strain rate in (2.4) is proportional to $\mathbf{R}_{\alpha}^{\top} \overline{\boldsymbol{\tau}}$, rather than to $\overline{\boldsymbol{\tau}}$, the normal direction to the yield surface.

Assuming $\overline{\mathcal{P}} \neq 0$, equation (2.3) takes the form:

$$
G^{-1} \frac{\partial \bar{\tau}}{\partial \bar{t}}=\nabla \bar{v}-\left[\frac{\overline{\boldsymbol{\tau}} \cdot \nabla \bar{v}}{Y^{2} \cos \alpha}\right] \mathbf{R}_{\alpha}^{\top} \overline{\boldsymbol{\tau}}
$$

Taking the dot product of (2.5) with $\overline{\boldsymbol{\tau}}$, we see that this equation implies that $\partial_{\bar{t}}|\overline{\boldsymbol{\tau}}|=0$ : i.e., the stress evolves tangentially along the yield surface $\{|\overline{\boldsymbol{\tau}}|=Y\}$. Even though formula (2.5) remains meaningful for stresses of any magnitude, from (2.4), this equation applies only when $|\bar{\tau}|=Y$.

When $\overline{\mathcal{P}} \neq 0$, material is said to be evolving plastically; otherwise elastically. For elastic deformations $(2.2,2.3)$ is equivalent to the wave equation with wave speed $\sqrt{G / \rho}$. By contrast, as follows from [7], $(2.2,2.5)$ for plastic deformations is illposed. Specifically, in some directions waves propagate as in a hyperbolic equation, but in other directions the wave speed is imaginary. In this paper we will discuss the ill-posedness of $(2.2,2.5)$ only in the context of one-dimensional solutions, in Section 2.3 below.

For much of this paper we shall deal with plastic deformations, so that (2.5) will be the constitutive relation. The reason for this restriction is that we shall be 
studying small perturbations of steady state solutions; all non-trivial steady-states of $(2.2,2.3)$ are everywhere-plastic. Furthermore, we now show that non-plastic perturbations decay rapidly to return the solution to a plastic state. To see this, consider an equilibrium solution $\bar{v}^{(0)}, \overline{\boldsymbol{\tau}}^{(0)}$ of $(2.2,2.3)$ that satisfies

$$
\overline{\boldsymbol{\tau}}^{(0)} \cdot \nabla \bar{v}^{(0)}>0, \quad \overline{\boldsymbol{\tau}}^{(0)} \cdot \overline{\boldsymbol{\tau}}^{(0)} \equiv Y^{2},
$$

everywhere, and examine the evolution of an infinitesimal linear perturbation of (2.6), $\bar{v}=\bar{v}^{(0)}+\varepsilon \bar{v}^{(1)}, \overline{\boldsymbol{\tau}}=\overline{\boldsymbol{\tau}}^{(0)}+\varepsilon \overline{\boldsymbol{\tau}}^{(1)}$, as $\varepsilon \rightarrow 0$. The perturbed solution is locally elastic if

$$
\eta \equiv \overline{\boldsymbol{\tau}}^{(0)} \cdot \overline{\boldsymbol{\tau}}^{(1)}<0 .
$$

For $\eta<0$, equation (2.3) with $\overline{\mathcal{P}}=0$ shows that $\eta$ evolves according to

$$
\frac{\partial \eta}{\partial \bar{t}}=\frac{G}{\varepsilon}\left(\overline{\boldsymbol{\tau}}^{(0)} \cdot \nabla \bar{v}^{(0)}\right)+O(1)>0 .
$$

From (2.6), the first term dominates, so we see that $\eta$ decays to zero in a finite, short $O(\varepsilon)$ time, and subsequent evolution will be plastic. Moreover, further justification for the restriction to plastic deformations will be given in Section 5 .

\subsection{Nondimensionalization}

We consider $(2.2,2.5)$ in the domain between two parallel plates; thus, we assume that $0<\bar{x}<L,-\infty<\bar{y}<\infty$. The parameter $L$ sets the scale for length. All motion is in the $\bar{z}$-direction, parallel to the plates. We shall study a problem in which the boundary conditions impose a strain rate of the order of magnitude $U / L$, where $U$ is a characteristic velocity. Combining these with the parameters from the equations of motion, we have the following list:

$$
\begin{array}{ll}
Y, G & \text { force/length } \\
\hline & ; \\
\rho & \text { mass } / \text { length }^{3} ; \\
L & \text { length; } \\
U & \text { length/time. }
\end{array}
$$

We define dimensionless variables

$$
x=\frac{\bar{x}}{L}, \quad y=\frac{\bar{y}}{L}, \quad t=\frac{\bar{t}}{T}, \quad v=\frac{\bar{v}}{U}, \quad \boldsymbol{\tau}=\frac{\bar{\tau}}{Y},
$$

where we choose the time scale $T$ to be

$$
T=\frac{\rho U L}{Y} .
$$

With these definitions, equations $(2.2,2.5)$ become

$$
\begin{aligned}
\frac{\partial v}{\partial t} & =\nabla \cdot \boldsymbol{\tau} \\
\mathcal{E}^{-1} \frac{\partial \boldsymbol{\tau}}{\partial t} & =\nabla v-\left[\frac{\boldsymbol{\tau} \cdot \nabla v}{\cos \alpha}\right] \mathbf{R}_{\alpha}^{\top} \boldsymbol{\tau}
\end{aligned}
$$


where

$$
\mathcal{E}=\left(\frac{G}{Y} \cdot \frac{U}{\sqrt{G / \rho}}\right)^{2},
$$

and the yield condition becomes $|\boldsymbol{\tau}| \equiv 1$. The time scale $T$ has been chosen so that only one dimensionless parameter $\mathcal{E}$ remains in the problem.

We note that $\mathcal{E}$ can be written in terms of the two dimensionless groups

$$
\frac{G}{Y}, \quad \text { and } \quad \frac{U}{\sqrt{G / \rho}}
$$

The first ratio $G / Y$ is moderately large, on the order of 50, in the biaxial experiments described in [12]. The second ratio, which is analogous to the Mach number in aerodynamics, is typically very small, since $U$ is small and the elastic wave speed $\sqrt{G / \rho}$ is large for all but the softest of materials. Therefore, $\mathcal{E}$ is small. Incidentally, note that the time scale $T$ may be rewritten

$$
T=\sqrt{\mathcal{E}} \frac{L}{\sqrt{G / \rho}}
$$

Thus, $T$ is short compared to $L / \sqrt{G / \rho}$, the time for a sound wave to cross the domain.

To make the connection with the companion paper [6], we now show that the problem studied there corresponds to the limit $\mathcal{E} \rightarrow \infty$. This limit is unphysical for granular flow, but, as described in [6], it occurs in other contexts. We note from $(2.10 \mathrm{~b})$ that, in this limit, the time scale for the evolution of the stress is fast. Reflecting this fact, suppose that the stress is in quasi-static equilibrium. Then, balancing the two terms on the right hand side of (2.10b) and using the yield condition for plasticity, $|\boldsymbol{\tau}|=1$, we obtain

$$
\boldsymbol{\tau}=\mathbf{R}_{\alpha} \frac{\nabla v}{|\nabla v|}
$$

Substituting into (2.10a) produces the nonlinear parabolic equation studied in [6]

$$
\frac{\partial v}{\partial t}=\nabla \cdot\left(\mathbf{R}_{\alpha} \frac{\nabla v}{|\nabla v|}\right)
$$

\subsection{Continuum equations in one space dimension}

In this section we will study the dynamics of one-dimensional solutions of (2.10). A trivial equilibrium solution is

$$
v_{\text {unif }}(x, y)=a x+b y, \quad \boldsymbol{\tau}_{\text {unif }}=\mathbf{R}_{\alpha}\left(\begin{array}{c}
a \\
b
\end{array}\right),
$$


where $a^{2}+b^{2}=1$. In this solution, material undergoes steady, uniform, plastic shear. We shall seek solutions of (2.10) in which the (nondimensionalized) velocity has the form

$$
v(x, y, t)=a x+b y+w(x, t),
$$

and the stress $\boldsymbol{\tau}=(p, q)^{\top}$ is independent of $y$. Thus, $w$ measures the departure of the velocity from the uniform shear solution. If the stress is independent of $y$ then $w$ is constrained to be independent of $y$ as well. Substituting the velocity gradient $\nabla v=\left(a+\partial_{x} w, b\right)^{\top}$ into the two-dimensional equations yields

$$
\begin{aligned}
\frac{\partial w}{\partial t} & =\frac{\partial p}{\partial x} \\
\mathcal{E}^{-1} \frac{\partial}{\partial t}\left(\begin{array}{c}
p \\
q
\end{array}\right) & =\left(\begin{array}{c}
a+\partial_{x} w \\
b
\end{array}\right)-\frac{1}{\cos \alpha}\left[\left(\begin{array}{c}
p \\
q
\end{array}\right) \cdot\left(\begin{array}{c}
a+\partial_{x} w \\
b
\end{array}\right)\right] \mathbf{R}_{\alpha}^{\top}((\underset{q}{2} .1) \mathrm{bb})
\end{aligned}
$$

where $p^{2}+q^{2} \equiv 1$. We consider (2.16) subject to periodic boundary conditions:

$$
w(1, t)=w(0, t), \quad \boldsymbol{\tau}(1, t)=\boldsymbol{\tau}(0, t) .
$$

Periodic boundary conditions are chosen for convenience of analysis; the precise boundary condition are expected to have little effect on the results.

In order to demonstrate ill-posedness in (2.16), let us linearize this equation about the steady state solution (2.14). Making the substitution $w=\tilde{w}, \boldsymbol{\tau}=\boldsymbol{\tau}_{\text {unif }}+\tilde{\boldsymbol{\tau}}$, where $\tilde{w}, \tilde{\tau}$ are assumed small, we obtain

$$
\begin{aligned}
\frac{\partial \tilde{w}}{\partial t} & =\frac{\partial \tilde{p}}{\partial x} \\
\mathcal{E}^{-1} \frac{\partial \tilde{\boldsymbol{\tau}}}{\partial t} & =\left[\left(\begin{array}{l}
1 \\
0
\end{array}\right)-\frac{p_{\text {unif }}}{\cos \alpha} \nabla v_{\text {unif }}\right] \frac{\partial \tilde{w}}{\partial x}-\frac{\nabla v_{\text {unif }}}{\cos \alpha}\left(\nabla v_{\text {unif }} \cdot \tilde{\boldsymbol{\tau}}\right)-\mathbf{R}_{\alpha}^{\top} \tilde{\boldsymbol{\tau}}(2.18 \mathrm{~b})
\end{aligned}
$$

where, to compactify the notation in (2.18), we write $\nabla v_{\text {unif }}=(a, b)^{\top}$. Solutions of these equations automatically satisfy

$$
\tilde{\boldsymbol{\tau}} \cdot \boldsymbol{\tau}_{\text {unif }}=0 .
$$

This is to be expected since solutions of the full equations (2.16) satisfy the yield condition $|\boldsymbol{\tau}|=1$, and (2.19) is the linearization of the yield condition.

We may use the linearized yield condition (2.19) to eliminate $\tilde{q}$ from (2.18). This calculation is simplified if we represent the underlying velocity gradient in terms of an angle (the minus sign is convenient below),

$$
\nabla v_{\text {unif }}=(a, b)^{\top}=(\cos (-\mu), \sin (-\mu))^{\top} .
$$

According to (2.14),

$$
\boldsymbol{\tau}_{\text {unif }}=(\cos (-\mu+\alpha), \sin (-\mu+\alpha))^{\top}=(\cos (\mu-\alpha),-\sin (\mu-\alpha))^{\top} .
$$


Therefore, by (2.19), $\tilde{\boldsymbol{\tau}}$ can be represented in terms of $\tilde{p}$ as

$$
\tilde{\boldsymbol{\tau}}=\frac{\tilde{p}}{\sin (\mu-\alpha)}\left(\begin{array}{c}
\sin (\mu-\alpha) \\
\cos (\mu-\alpha)
\end{array}\right) \text {. }
$$

Substituting this expression into (2.18b), making use of (2.18a), and applying several trigonometric addition formulas, we find that $\tilde{p}$ satisfies

$$
\mathcal{E}^{-1} \frac{\partial^{2} \tilde{p}}{\partial t^{2}}=\frac{\sin (\mu-\alpha) \sin \mu}{\cos \alpha} \frac{\partial^{2} \tilde{p}}{\partial x^{2}}-\frac{1}{\cos \alpha} \frac{\partial \tilde{p}}{\partial t} .
$$

If the coefficient of the $\partial_{x x} \tilde{p}$ term is positive, then (2.21) is a damped wave equation. On the other hand, if

$$
0<\mu<\alpha
$$

then this coefficient is negative, and so (2.21), and hence system (2.18), is ill-posed.

Remark: For the two-dimensional problem $(2.2,2.5)$, this equilibrium-given by (2.14) in dimensionless variables - is always ill-posed. Indeed, as proved in [7], a plane-wave perturbation $e^{i\left(k_{1} x+k_{2} y\right)}$ suffers catastrophic amplification if the direction of propagation $\mathbf{k}$ lies in the sector

$$
\arg (a, b)^{\top}<\arg \mathbf{k}<\arg (a, b)^{\top}+\alpha
$$

where $\arg (a, b)^{\top}=\arctan (b / a)$. In restricting ourselves to solutions of $(2.2,2.5)$ of the form (2.15), we are allowing only plane waves that propagate parallel to the $x$-axis. The one-dimensional problem (i.e. $k_{2}=0$ ) is ill-posed if and only if the $x$-axis lies in the wedge of ill-posed directions of the two-dimensional problem.

In analyzing non-trivial, shear-band equilibria in Section 3, we shall assume that the underlying velocity gradient $(a, b)^{\top}$ satisfies

$$
a>0, \quad 0<-b / a<\tan \alpha .
$$

On recalling (2.20) and (2.22), we see that (2.23) implies that the one-dimensional continuum equations (2.16) are ill-posed at the trivial solution (2.14).

\subsection{Discretization of the one-dimensional equations}

We will apply a finite-difference discretization on the interval $0 \leq x \leq 1$; we define $N+1$ equally-spaced grid points $x_{n}=n \delta$ for $n=0,1, \ldots, N$, where $\delta=1 / N$. Let $w_{n}(t)$ be an approximation for $w\left(x_{n}, t\right)$, the velocity at the grid points. As illustrated in Figure 2, we evaluate the stress on a staggered grid: let $\boldsymbol{\tau}_{n}(t)=\left(p_{n}(t), q_{n}(t)\right)$ be an approximation for $\boldsymbol{\tau}\left(x_{n+\frac{1}{2}}, t\right)$.

To discretize (2.16), we replace derivatives by finite differences. If $\Delta$ is the 


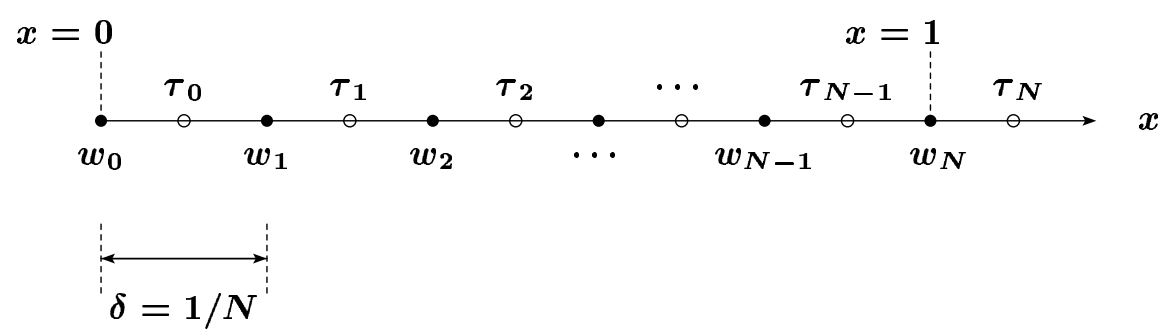

Figure 2: The staggered grid used for the velocity and stress components.

forward difference operator $\Delta w_{n} \equiv w_{n+1}-w_{n}$, then the discretization is

$$
\begin{array}{rlr}
\frac{d w_{n}}{d t}= & \Delta p_{n-1} / \delta & n=1,2, \ldots, N \text { (2.24a) } \\
\mathcal{E}^{-1} \frac{d \boldsymbol{\tau}_{n}}{d t}= & \left(\begin{array}{c}
a+\Delta w_{n} / \delta \\
b
\end{array}\right) & \\
& -\left[\frac{p_{n}\left[a+\Delta w_{n} / \delta\right]+q_{n} b}{\cos \alpha}\right] \mathbf{R}_{\alpha}^{\top} \boldsymbol{\tau}_{n} & n=0,1, \ldots, N-12.24 \mathrm{~b})
\end{array}
$$

This system of coupled ODE provides a formally second-order-accurate approximation for the PDE (2.16).

Since $\boldsymbol{\tau}_{n}$ has two components for each $n$, equations (2.24) are a system of $3 N$ differential equations for $3(N+1)$ unknowns. Periodic boundary conditions,

$$
w_{N}=w_{0}, \quad \tau_{N}=\tau_{0},
$$

provide the additional three equations needed to complete the system. Equivalently, we may say that (2.25) eliminates three of the unknowns in (2.24). It is natural to eliminate $\boldsymbol{\tau}_{N}$ because as may be seen from Figure $3, \boldsymbol{\tau}_{N}$ approximates the stress at a point outside the basic period interval $0<x<1$. It will be convenient to eliminate $w_{0}$ rather than $w_{N}$. Note that the indexing in (2.24) is arranged so that the time derivatives of $w_{0}$ and $\boldsymbol{\tau}_{N}$ do not appear in the equations.

Remark: If the yield condition is invoked, the counting changes. Specifically, one may discard one component of (2.24b) in favor of the yield condition $\left|\boldsymbol{\tau}_{n}\right|=$ $1, n=0,1, \ldots, N-1$. In this case we have a system of $2 N$ differential equations plus $N$ algebraic equations for $3 N$ unknowns. (Note that the yield condition at the right boundary, $\left|\boldsymbol{\tau}_{N}\right|=1$, is not an independent equation since it is a consequence of $\left|\boldsymbol{\tau}_{0}\right|=1$ and periodic boundary conditions.) Although from this point of view the system looks like a differential-algebraic system, in fact, as we shall see below, the algebraic equations can be used to eliminate $N$ of the unknowns, resulting in a system of $2 N$ differential equations for $2 N$ unknowns. 

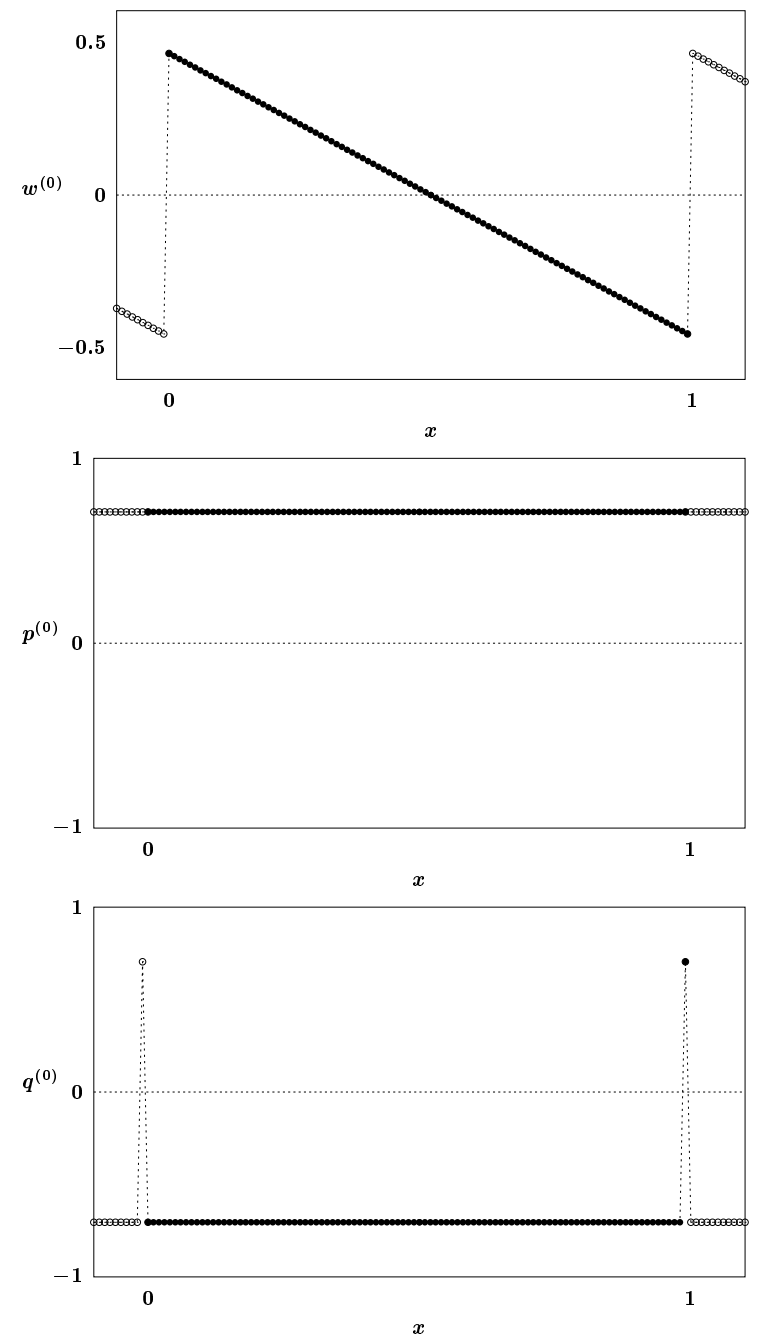

Figure 3: Profiles of $w, p, q$ for the equilibrium shear band solution (the periodic extensions outside $0<x<1$ shown with open circles). 


\section{Formulation of the linear stability problem}

\subsection{Equilibria of the discrete model with one shear band}

To derive an equilibrium equation from (2.24), let us, as in the previous remark, discard one component of (2.24b) and invoke the yield condition $|\boldsymbol{\tau}|=1$ instead. This yields

$$
\begin{aligned}
\Delta p_{n} & =0 \\
p_{n}^{2}+q_{n}^{2} & =1 \\
\arg \boldsymbol{\tau}_{n}-\alpha & =\arg \left(\begin{array}{c}
a+\Delta w_{n} / \delta \\
b
\end{array}\right)
\end{aligned}
$$

for $n=0,1, \ldots, N-1$. Here $\arg (p, q)^{\top}$ refers to the argument, $\tan ^{-1}(q / p)$; thus, we are discarding the radial component of the vector equation $(2.24 \mathrm{~b})$ and retaining the angular component.

According to (3.1a), $p_{n}$ is independent of $n$; since $\left|p_{n}\right| \leq 1$, we may write $p_{n}=\cos \phi, n=0,1, \ldots, N-1$ for some angle $\phi$. From (3.1b),

$$
q_{n}= \pm \sin \phi, \quad n=0,1, \ldots, N-1 .
$$

There are many different choices for the plus/minus signs in (3.2), each leading to a different steady-state solution of (2.24) (cf. [6]). In this paper we focus on singleshear-band equilibria, by which we mean a case in which there is exactly one entry in (3.2) with the positive sign. Without loss of generality we may assume that the positive sign occurs for $n=0$. Thus we have

$$
q_{0}=+\sin \phi ; \quad q_{n}=-\sin \phi, \quad n=1,2, \ldots, N-1,
$$

where, invoking (2.23), we will show that $\phi>0$.

According to (3.1c) and (3.3), $\Delta w_{n}$ has a common value for all $n \neq 0$, which we will denote by $\Delta w_{+}$. Thus, the collection of $N$ equations (3.1c) is equivalent to the pair of equations

$$
\begin{aligned}
\tan (\phi-\alpha) & =\frac{b}{a+\Delta w_{0} / \delta} \\
\tan (-\phi-\alpha) & =\frac{b}{a+\Delta w_{+} / \delta} .
\end{aligned}
$$

The two equations (3.4) contain three unknowns: $\phi, \Delta w_{0}$, and $\Delta w_{+}$. To complete this system, we invoke the boundary conditions (2.25). Although the stress boundary conditions are trivial, periodicity for the velocity yields the restriction that

$$
0=w_{N}-w_{0}=\sum_{n=0}^{N-1} \Delta w_{n}=\Delta w_{0}+(N-1) \Delta w_{+} .
$$


Recalling that $N=1 / \delta$, we rewrite this equation as

$$
\Delta w_{+}=-\frac{\delta}{1-\delta} \Delta w_{0}=-\delta \Delta w_{0}+O\left(\delta^{2}\right)
$$

It suffices for our purposes to analyze $(3.4,3.5)$ only in the limit $\delta \rightarrow 0$. To leading order in this limit, (3.4a) reduces to $\phi=\alpha$. Similarly, substituting $\phi=\alpha$ into (3.4b) and recalling (3.5), we obtain

$$
\Delta w_{0}=a+b \cot 2 \alpha+O(\delta) .
$$

Note that $\Delta w_{0}=O(1)$, while by (3.5), $\Delta w_{+}=O(\delta)$. The velocity and stress profiles of a typical solution are sketched in Figure 3. We refer to the large jump in the velocity between grid points $n=0$ and $n=1$ as a shear band.

Below we shall need the $O(\delta)$-correction to the leading-order solution for $\phi$. Substituting (3.6) into (3.4a) and using the small-angle approximation for $\tan (\phi-$ $\alpha)$, we deduce that

$$
\phi=\alpha+[a / b+\cot 2 \alpha]^{-1} \delta+O\left(\delta^{2}\right) .
$$

For small $\delta$, it is easily established from the implicit function theorem that (3.4, 3.5) has a unique solution for $\phi, \Delta w_{0}, \Delta w_{+}$. However, the solution of (3.1) is not unique since the equations depend only on differences of the velocity, $\Delta w_{n}$; i.e., the velocity $w_{n}$ is determined only up to an additive constant. Thus, even after the location of the shear band has been fixed, $(2.24,2.25)$ admits a one-parameter family of single-shear-band equilibria.

Finally, note that the one-shear-band equilibria satisfy both conditions in (2.4) for plastic deformation: by (3.1b) we have the yield condition, and since from (3.1c)

$$
\left(\begin{array}{c}
a+\Delta w_{n} / \delta \\
b
\end{array}\right) \cdot \boldsymbol{\tau}_{n}=\left|\left(\begin{array}{c}
a+\Delta w_{n} / \delta \\
b
\end{array}\right)\right| \cos \alpha>0,
$$

we also have the loading condition.

\subsection{The eigenvalue problem for linearized stability}

Assuming (2.23) holds, let $w^{(0)}, \boldsymbol{\tau}^{(0)}$ be a single-shear-band equilibrium solution of the discretized equations $(2.24,2.25)$, as constructed above. Thus,

$$
\begin{gathered}
w_{n}^{(0)}=w_{1}^{(0)}+(n-1) \Delta w_{+}, \quad n=1,2, \ldots, N-1, \quad w_{0}^{(0)}=w_{1}^{(0)}+(N-1) \Delta w_{+}, \\
\boldsymbol{\tau}_{n}^{(0)}=\left(\begin{array}{c}
\cos \phi \\
-\sin \phi
\end{array}\right), \quad n=1,2, \ldots, N-1, \quad \boldsymbol{\tau}_{0}^{(0)}=\left(\begin{array}{c}
\cos \phi \\
\sin \phi
\end{array}\right) .
\end{gathered}
$$

where $w_{1}^{(0)}$ is arbitrary, $\phi$ is given by (3.7), and the velocity differences $\Delta w_{+}$are given by $(3.5,3.6)$. To analyze the stability of this equilibrium with respect to perturbation, we let

$$
w_{n}(t)=w_{n}^{(0)}+\tilde{w}_{n}(t), \quad \boldsymbol{\tau}_{n}(t)=\boldsymbol{\tau}_{n}^{(0)}+\tilde{\boldsymbol{\tau}}_{n}(t),
$$


substitute (3.9) into (2.24), and linearize. This calculation is similar to the derivation of (2.18) in Section 2.3. The fact that space has been discretized in (2.24) does not significantly change this calculation. However, the present derivation is longer because here the unperturbed velocity $w^{(0)}$ is non-vanishing and the unperturbed stress $\boldsymbol{\tau}^{(0)}$ depends on position. As in Section 2.3, the linearized yield condition

$$
\boldsymbol{\tau}_{n}^{(0)} \cdot \tilde{\boldsymbol{\tau}}_{n}(t)=0, \quad n=0,1, \ldots, N-1
$$

may be invoked to eliminate $\tilde{q}_{n}$, the second component of $\tilde{\boldsymbol{\tau}}_{n}$. This procedure leads to a system of equations of the form

$$
\begin{aligned}
\frac{d \tilde{w}_{n}}{d t} & =\left(\tilde{p}_{n}-\tilde{p}_{n-1}\right) / \delta, & & n=1,2, \ldots, N \\
\mathcal{E}^{-1} \frac{d \tilde{p}_{n}}{d t} & =A\left(\tilde{w}_{n+1}-\tilde{w}_{n}\right) / \delta-B \tilde{p}_{n}, & & n=1,2, \ldots, N-1 \\
\mathcal{E}^{-1} \frac{d \tilde{p}_{0}}{d t} & =-C\left(\tilde{w}_{1}-\tilde{w}_{0}\right)-D \tilde{p}_{0} / \delta & & (n=0)
\end{aligned}
$$

where $A, B, C$, and $D$ are positive constants that depend on the parameters $\alpha, a, b$, and $\delta$. Since they depend on $a$ and $b$ only through the ratio $b / a$, we write $b / a=$ $-\tan \mu$ as in (2.20). In the limit $\delta \rightarrow 0$, these parameters are given by

$$
\begin{array}{ll}
A=2 \sin ^{2} \alpha+O(\delta) & B=\frac{\sin \mu}{\cos \alpha \sin (2 \alpha)}+O(\delta) \\
C=\frac{\tan \alpha}{\cot \mu-\cot 2 \alpha}+O(\delta) & D=\frac{\sin \mu}{\cos \alpha}(\cot \mu-\cot 2 \alpha)+O(\delta) .
\end{array}
$$

Of course (3.11) must be supplemented with periodic boundary conditions.

The structure of equations (3.11) is noteworthy. In the bulk of the interval, equations (3.11) represent the discretization of a wave equation with a dissipative term. However, at the exceptional point, $n=0$, (i) the coefficient of $\Delta \tilde{w}_{0}$ has the opposite sign and is reduced by a factor of $\delta$ in magnitude, and (ii) the coefficient of $\tilde{p}_{0}$, the dissipative term, is larger by a factor of $1 / \delta$. Only the term $-C \Delta \tilde{w}_{0}$ acts against stability; however, depending on the magnitude of $\mathcal{E}$ relative to $\delta$, this term may in fact destabilize (3.11).

We seek solutions of (3.11) of the form $\tilde{w}_{n}(t)=e^{\lambda t} W_{n}, \tilde{p}_{n}(t)=e^{\lambda t} P_{n}$, subject to periodic boundary conditions

$$
W_{N}=W_{0}, \quad P_{N}=P_{0} .
$$

This ansatz leads to the eigenvalue problem

$$
\begin{aligned}
\lambda W_{n} & =\left(P_{n}-P_{n-1}\right) / \delta, & n=1,2, \ldots, N \\
\mathcal{E}^{-1} \lambda P_{n} & =A\left(W_{n+1}-W_{n}\right) / \delta-B P_{n}, & n=1,2, \ldots, N-1 \\
\mathcal{E}^{-1} \lambda P_{0} & =-C\left(W_{1}-W_{0}\right)-D P_{0} / \delta & (n=0) .
\end{aligned}
$$


If the boundary conditions (3.13) are used to eliminate $W_{0}$ and $P_{N}$ from (3.14), we are left with a $2 N$-dimensional eigenvalue problem. We shall see that (3.14) always has one zero eigenvalue, corresponding to the fact that there is a 1-parameter family of equilibria. The equilibrium solution $w^{(0)}, \boldsymbol{\tau}^{(0)}$ will be stable if the remaining $2 N-1$ eigenvalues lie in the stable half plane, $\{\lambda \in \mathbb{C}: \operatorname{Re}(\lambda)<0\}$.

\section{Perturbation theory estimate of the linear sta- bility condition}

We shall calculate the eigenvalues of (3.14) in the asymptotic limit $\delta \rightarrow 0$. Specifically, we shall show that for small $\delta$, if

$$
\mathcal{E}>\left[\frac{2 \sin ^{2}(2 \alpha)}{\sin (2 \alpha-\mu)}\right]^{2} \delta+O\left(\delta^{2}\right)
$$

then all nonzero eigenvalues of (3.14) lie in the stable half plane.

The complete proof of this result is rather technical for the following reason. The dimension of this problem is proportional to $1 / \delta$. Thus, if the eigenvalues $\lambda_{k}$ of (3.14) are enumerated by $k$, this index has the range

$$
k=1,2, \ldots, O(1 / \delta),
$$

which increases without bound as $\delta \rightarrow 0$. Although it is relatively easy to estimate $\lambda_{k}$ for any fixed $k$, it is difficult to obtain estimates that are uniformly valid over the entire range (4.2), as is needed to accurately describe the entire spectrum and prove stability.

In this section we begin the calculation with perturbation-theory estimates for the eigenvalues. Although these preliminary estimates are not uniform, they provide invaluable guidance for the full calculation that follows in the next section. As it happens, for large $k$, the actual eigenvalues are more stable than the perturbation theory prediction, and in fact the correct stability condition (4.1) does emerge from this calculation.

\section{(a) Formulation of (3.14) as a perturbation-theory calculation}

Let us write the matrix (3.14) in block form. Using (3.13) to eliminate $W_{0}$ and $P_{N}$ from (3.14), we represent an eigenvector $\mathbf{V} \in \mathbb{C}^{2 N}$ in the form

$$
\mathbf{V}=\left(\begin{array}{c}
\mathbf{P} \\
\mathbf{W}
\end{array}\right)=\left(\begin{array}{c}
P_{0} \\
\mathbf{P}_{+} \\
\mathbf{W}
\end{array}\right)
$$

where $\mathbf{W}=\left(W_{1}, \ldots, W_{N}\right)^{\top}, \mathbf{P}=\left(P_{0}, P_{1}, \ldots, P_{N-1}\right)^{\top}$, and $\mathbf{P}_{+}=\left(P_{1}, \ldots, P_{N-1}\right)^{\top}$. In this subsection, in forming the vector $\mathbf{V}$, we put $\mathbf{P}$ before $\mathbf{W}$ because it is convenient 
to have $P_{0}$, which requires special treatment, as the first component. Reflecting this reversal of order, we begin by rewriting equation (3.14c) in this notation as

$$
-\delta^{-1} D \mathcal{E} P_{0}-C \mathcal{E} \mathbf{Z}^{\top} \mathbf{W}=\lambda P_{0},
$$

where $\mathbf{Z} \in \mathbb{R}^{N}$ is defined by

$$
\mathbf{Z}=(1,0, \ldots, 0,-1)^{\top}
$$

Similarly, (3.14b) may be rewritten as

$$
-B \mathcal{E} \mathbf{P}_{+}+\delta^{-1} A \mathcal{E} \Delta \mathbf{W}=\lambda \mathbf{P}_{+}
$$

where $\boldsymbol{\Delta}$ is the $(N-1) \times N$ matrix representing the forward difference operator,

$$
\Delta=\left(\begin{array}{rrrrrrrr}
-1 & 1 & & & & & \\
& -1 & 1 & & & & \\
& & \cdot & \cdot & & & \\
& & & \cdot & \cdot & & \\
& & & & \cdot & \cdot & \\
& & & & & -1 & 1
\end{array}\right)
$$

Finally, equation (3.14a) may be written in matrix notation as

$$
-\delta^{-1}\left(\begin{array}{r|rrrrrr}
1 & -1 & & & & \\
& 1 & -1 & & & & \\
& & 1 & \cdot & & & \\
& & & \cdot & \cdot & & \\
& & & & \cdot & \cdot & \\
-1 & & & & & & -1 \\
& & & & & & 1
\end{array}\right)\left(\begin{array}{c}
P_{0} \\
\mathbf{P}_{+}
\end{array}\right)=\lambda \mathbf{W}
$$

on partitioning this matrix into two blocks corresponding to $\left(P_{0}, \mathbf{P}_{+}\right)$, we rewrite this as

$$
-\delta^{-1} \mathbf{Z} P_{0}-\delta^{-1} \boldsymbol{\Delta}^{\top} \mathbf{P}_{+}=\lambda \mathbf{W}
$$

where $\boldsymbol{\Delta}^{\top}$ is the transpose of (4.3), an $N \times(N-1)$ matrix.

In summary, we have rewritten the eigenvalue problem (3.14) in the block matrix form

$$
\left(\begin{array}{ccc}
-\delta^{-1} D \mathcal{E} & 0 & -C \mathcal{E} \mathbf{Z}^{\top} \\
0 & -B \mathcal{E} \mathbf{I} & \delta^{-1} A \mathcal{E} \boldsymbol{\Delta} \\
-\delta^{-1} \mathbf{Z} & -\delta^{-1} \boldsymbol{\Delta}^{\top} & \mathbf{0}
\end{array}\right)\left(\begin{array}{c}
P_{0} \\
\mathbf{P}_{+} \\
\mathbf{W}
\end{array}\right)=\lambda\left(\begin{array}{c}
P_{0} \\
\mathbf{P}_{+} \\
\mathbf{W}
\end{array}\right)
$$

where in the 2, 2-entry of this matrix $\mathbf{I}$ is the $(N-1) \times(N-1)$ identity matrix.

The matrix in (4.4) can be written in the form $\mathcal{M}=\delta^{-1}\left(\mathcal{M}_{(0)}+\delta \mathcal{M}_{(1)}\right)$, where

$$
\mathcal{M}_{(0)}=\left(\begin{array}{c|cc}
-D \mathcal{E} & 0 & \mathbf{0} \\
\hline 0 & \mathbf{0} & A \mathcal{E} \boldsymbol{\Delta} \\
-\mathbf{Z} & -\boldsymbol{\Delta}^{\top} & \mathbf{0}
\end{array}\right)
$$


and

$$
\mathcal{M}_{(1)}=\left(\begin{array}{ccc}
0 & 0 & C \mathcal{E} \mathbf{Z}^{\top} \\
0 & -B \mathcal{E} \mathbf{I} & \mathbf{0} \\
\mathbf{0} & \mathbf{0} & \mathbf{0}
\end{array}\right)
$$

Note that the subscripts (0), (1) refer to the leading order matrix and the first perturbation as $\delta \rightarrow 0$; they are unrelated to subscripts labeling grid points.

To calculate the eigenvalues of $\mathcal{M} \mathbf{V}=\lambda \mathbf{V}$ in the limit $\delta \rightarrow 0$, we apply perturbation theory. According to this theory (cf. [13] p. 323), if $\lambda_{(0)}$ is a simple eigenvalue of $\delta^{-1} \mathcal{M}_{(0)}$, then $\mathcal{M}$ has a corresponding eigenvalue given, to lowest order, by

$$
\lambda \sim \lambda_{(0)}+\frac{\left\langle\mathbf{V}^{\dagger}, \mathcal{M}_{(1)} \mathbf{V}\right\rangle}{\left\langle\mathbf{V}^{\dagger}, \mathbf{V}\right\rangle}
$$

where $\mathbf{V}$ is the eigenvector of $\delta^{-1} \mathcal{M}_{(0)}$ for $\lambda_{(0)}$ and $\mathbf{V}^{\dagger}$ is the associated adjoint eigenvector, i.e., the nullvector of $\left(\delta^{-1} \mathcal{M}_{(0)}^{\top}-\bar{\lambda}_{(0)} \mathbf{I}\right)$, with $\bar{\lambda}_{(0)}$ denoting the complex conjugate of $\lambda_{(0)}$. (Since $\mathcal{M}_{(0)}$ has real entries, its transpose equals its Hermitian adjoint.) The brackets in (4.7) denote a scaled inner product on $\mathbb{C}^{2 N}$,

$$
\left\langle\left(\begin{array}{c}
P_{0} \\
\mathbf{P}_{+} \\
\mathbf{W}
\end{array}\right),\left(\begin{array}{c}
Q_{0} \\
\mathbf{Q}_{+} \\
\mathbf{X}
\end{array}\right)\right\rangle=\delta\left[\overline{P_{0}} Q_{0}+\sum_{j=1}^{N-1} \overline{P_{j}} Q_{j}+\sum_{j=1}^{N} \overline{W_{j}} X_{j}\right] .
$$

\section{(b) The eigenvectors to leading order}

Two eigenvalues of $\delta^{-1} \mathcal{M}_{(0)}$ may be determined by inspection:

$$
\text { (a) } \lambda=-D \mathcal{E} / \delta \quad \text { and } \quad \text { (b) } \lambda=0 \text {. }
$$

As may be seen from the block-triangular structure of $\mathcal{M}_{(0)}$, the first eigenvalue is associated with the 1,1-entry of $\boldsymbol{M}_{(0)}$. This eigenvalue, a consequence of the discretization, is far inside the stable half plane, and hence it is not vulnerable to becoming unstable under perturbation. The second eigenvalue, whose eigenvector has components $\mathbf{P}=\mathbf{0}$ and $\mathbf{W}=(1,1, \cdots, 1)^{\top}$, corresponds to the invariance of (3.14) with respect to uniform shifts of $\mathbf{W}$, i.e., $W_{n} \mapsto W_{n}+$ const. for all $n$.

We now focus on the $2 N-1$ eigenvalues of $\mathcal{M}_{(0)}$ not associated with the 1,1entry of $\mathcal{M}_{(0)}$. Because $\mathcal{M}_{(0)}$ is (block) lower triangular, any of these eigenvectors satisfies

$$
P_{0}=0 .
$$

Thus, the eigenvalue problem for $\delta^{-1} \mathcal{M}_{(0)}$ involves only the $\mathbf{P}_{+}, \mathbf{W}$-components, and it reduces to

$$
\begin{aligned}
\delta^{-1} A \mathcal{E} \boldsymbol{\Delta} \mathbf{W} & =\lambda \mathbf{P}_{+} \\
-\delta^{-1} \boldsymbol{\Delta}^{\top} \mathbf{P}_{+} & =\lambda \mathbf{W} .
\end{aligned}
$$

Writing this system in components, we obtain,

$$
\delta^{-1} A \mathcal{E}\left(W_{n+1}-W_{n}\right)=\lambda P_{n} \quad n=1,2, \cdots, N-1
$$



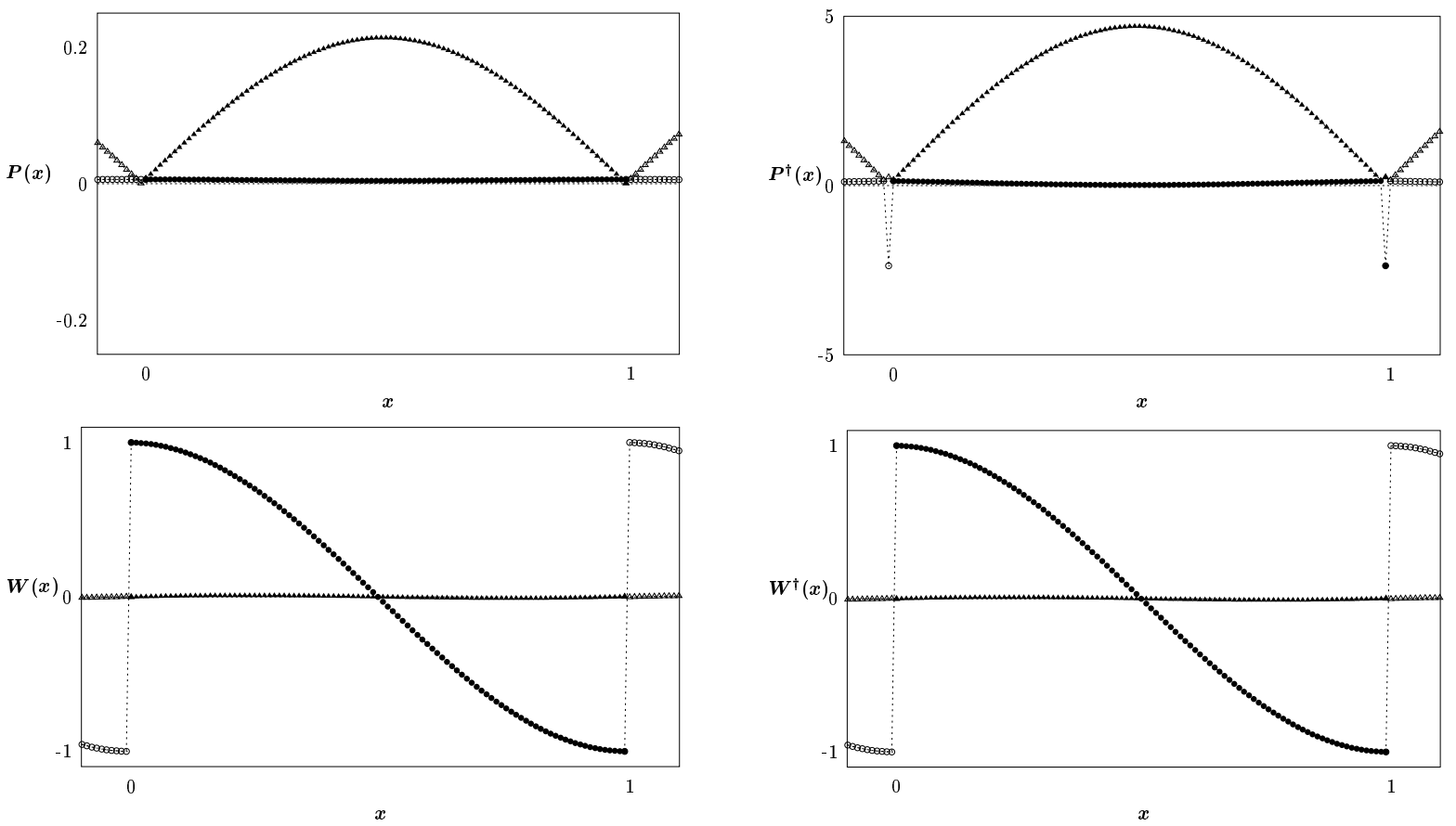

Figure 4: (Left column) The eigenfunctions $P(x), W(x)$ (4.15b) and (Right column) the adjoint eigenfunctions $P^{\dagger}(x), W^{\dagger}(x)$ (4.19) for $k=1$ calculated for $N=100$. Real part shown with circles, imaginary part shown with triangles (the periodic extensions shown with open symbols). 
and

$$
\begin{aligned}
\delta^{-1} P_{1} & =\lambda W_{1} \\
\delta^{-1}\left(P_{n}-P_{n-1}\right) & =\lambda W_{n} \quad n=2,3, \cdots, N-1 \\
-\delta^{-1} P_{N-1} & =\lambda W_{N} .
\end{aligned}
$$

Recalling from (4.10) that $P_{0}=0$ and that by periodicity $P_{N}=0$, we may rewrite these last equations in the unified form

$$
\delta^{-1}\left(P_{n}-P_{n-1}\right)=\lambda W_{n} \quad n=1,2, \cdots, N .
$$

In the limit $\delta \rightarrow 0$, we recognize equations (4.11) and (4.12) as a second-order accurate system of finite difference equations for the system of two ordinary differential equations for functions $P(x), W(x)$ on $0<x<1$,

$$
\begin{aligned}
A \mathcal{E} \frac{d W}{d x} & =\lambda P, \\
\frac{d P}{d x} & =\lambda W .
\end{aligned}
$$

As dictated by (4.10), we impose Dirichlet boundary conditions on these functions,

$$
P(0)=P(1)=0 .
$$

We use the solutions of this continuous problem to obtain convenient approximations for the underlying discrete systems. The solutions of $(4.13,4.14)$ are

$$
P(x)=i \sqrt{A \mathcal{E}} \sin (k \pi x), \quad W(x)=\cos (k \pi x),
$$

with eigenvalues,

$$
\lambda=i k \pi \sqrt{A \mathcal{E}}, \quad k=0, \pm 1, \pm 2, \cdots .
$$

Thus, for the eigenvalues and eigenvectors of $\delta^{-1} \mathcal{M}_{(0)}$, we have

$$
\begin{gathered}
\lambda_{(0)} \approx i k \pi \sqrt{A \mathcal{E}} \\
\mathbf{V}=\left(\begin{array}{c}
P_{0} \\
\mathbf{P}_{+} \\
\mathbf{W}
\end{array}\right) \approx \mathcal{R}\left(\begin{array}{c}
0 \\
i \sqrt{A \mathcal{E}} \sin (k \pi x) \\
\cos (k \pi x)
\end{array}\right), \quad k=0, \pm 1, \pm 2, \cdots,
\end{gathered}
$$

(see Figure 4) where the $\mathcal{R}$ operator projects continuous function onto the corresponding discrete representations of our staggered grid scheme, see Figure 2. We have indicated an infinite range for $k$ in $(4.15 \mathrm{~b})$ because for any fixed $k$, if $\delta$ is sufficiently small, $\delta^{-1} \mathcal{M}_{(0)}$ has an eigenvalue/eigenvector closely approximated by (4.15). Incidentally, the case $k=0$ in (4.15) repeats the zero eigenvalue already obtained, and it will be shown to be an exceptional case for the calculations below. Note that the eigenvalues (4.15a) of $\delta^{-1} \mathcal{M}_{(0)}$ are purely imaginary; thus stability 
will be determined by the real part of the correction term in (4.7), computed in the following two subsections.

\section{(c) The eigenvectors of the adjoint problem}

The adjoint eigenvector $\mathbf{V}^{\dagger}$ of $\delta^{-1} \mathcal{M}_{(0)}$ is the eigenvector of $\delta^{-1} \mathcal{M}_{(0)}^{\top}$ associated to the conjugate eigenvalue $\bar{\lambda}_{(0)}$. As in the previous calculation (b), the $\mathbf{P}_{+}^{\dagger}, \mathbf{W}^{\dagger}-$ components of the eigenvector may be approximated by an eigenfunction of the continuum problem for $P^{\dagger}(x), W^{\dagger}(x)$,

$$
\begin{aligned}
-\frac{d W^{\dagger}}{d x} & =\lambda P^{\dagger} \\
-A \mathcal{E} \frac{d P^{\dagger}}{d x} & =\lambda W^{\dagger}
\end{aligned}
$$

subject to the same boundary conditions, $P^{\dagger}(0)=P^{\dagger}(1)=0$. The solution of (4.16) associated with eigenvalue $\bar{\lambda}_{(0)}=-i \sqrt{A \mathcal{E}} k \pi$ is

$$
P^{\dagger}(x)=\frac{i}{\sqrt{A \mathcal{E}}} \sin (k \pi x), \quad W^{\dagger}(x)=\cos (k \pi x) .
$$

Unlike the calculation of the eigenvectors in subsection (b), the $P_{0}^{\dagger}$-component of $\mathbf{V}^{\dagger}$ need not vanish since $\mathcal{M}_{(0)}^{\top}$ is upper triangular. Indeed, we see from (4.5) that

$$
-\delta^{-1} D \mathcal{E} P_{0}^{\dagger}-\delta^{-1}\left(W_{1}^{\dagger}-W_{N}^{\dagger}\right)=\lambda P_{0}^{\dagger} .
$$

According to the continuum approximation (4.17) for $W_{1}^{\dagger}, W_{N}^{\dagger}$ in (4.18), we have

$$
W_{1}^{\dagger} \approx \lim _{x \rightarrow 0^{+}} W^{\dagger}(x)=1, \quad W_{N}^{\dagger} \approx \lim _{x \rightarrow 1^{-}} W^{\dagger}(x)=(-1)^{k} .
$$

Substituting these values into (4.18) yields

$$
P_{0}^{\dagger} \approx \frac{(-1)^{k}-1}{D \mathcal{E}+\delta \lambda^{(0)}} \sim \frac{(-1)^{k}-1}{D \mathcal{E}},
$$

where the final term gives the leading order value for $\delta \rightarrow 0$. In summary, we have obtained the adjoint eigenvector as

$$
\mathbf{V}^{\dagger}=\left(\begin{array}{c}
P_{0}^{\dagger} \\
\mathbf{P}_{+}^{\dagger} \\
\mathbf{W}^{\dagger}
\end{array}\right) \approx \mathcal{R}\left(\begin{array}{c}
{\left[(-1)^{k}-1\right] /(D \mathcal{E})} \\
i \sin (k \pi x) / \sqrt{A \mathcal{E}} \\
\cos (k \pi x)
\end{array}\right)
$$

We note that in the adjoint $\mathbf{V}^{\dagger}$, unlike for the eigenvector $\mathbf{V}$, the limit as $x \rightarrow 0$ of $P^{\dagger}(x)$ does not equal $P_{0}^{\dagger}$ (see Figure 4). This property is a consequence of the existence of the shear band in the equilibrium solution of $(2.24,2.25)$.

\section{(d) Completion of the calculation}


To evaluate (4.7), we need to adapt the inner product (4.8) to the continuum approximation for the $\mathbf{P}_{+}, \mathbf{W}$-components of the eigenvectors. Recognizing the second and third terms on the RHS of (4.8) as Riemann sums, we take

$$
\left\langle\left(\begin{array}{c}
P_{0} \\
\mathbf{P}_{+} \\
\mathbf{W}
\end{array}\right),\left(\begin{array}{c}
Q_{0} \\
\mathbf{Q}_{+} \\
\mathbf{X}
\end{array}\right)\right\rangle \approx \delta \overline{P_{0}} Q_{0}+\int_{0}^{1}[\overline{P(x)} Q(x)+\overline{W(x)} X(x)] d x .
$$

The $\overline{P_{0}} Q_{0}$-term in (4.20) must be handled separately since this component of the eigenvectors is not described by the continuum approximation.

Applying (4.6) to the right eigenvector (4.15b), we find

$$
\mathcal{M}_{(1)} \mathbf{V} \approx \mathcal{R}\left(\begin{array}{c}
C \mathcal{E}\left[(-1)^{k}-1\right] \\
-i B \mathcal{E} \sqrt{A \mathcal{E}} \sin (k \pi x) \\
\mathbf{0}
\end{array}\right)
$$

If $k=0$, which corresponds to the unperturbed eigenvalue $\lambda_{(0)}=0$, then $\mathcal{M}_{(1)} \mathbf{V}=$ $\mathbf{0}$; thus, according to (4.7), the corrected eigenvalue is still zero; in other words, the case $k=0$ leads to the zero eigenvalue already noted in (4.9). If $k \neq 0$, then taking the inner product with $\mathbf{V}^{\dagger}$ yields

$$
\left\langle\mathbf{V}^{\dagger}, \mathcal{M}_{(1)} \mathbf{V}\right\rangle=(\delta C / D)\left[(-1)^{k}-1\right]^{2}-B \mathcal{E} / 2=\left\{\begin{array}{cl}
4 \delta C / D-B \mathcal{E} / 2 & k \text { odd }, \\
-B \mathcal{E} / 2 & k \text { even }(k \neq 0) .
\end{array}\right.
$$

Regarding the denominator in (4.7), the eigenvectors were normalized so that $\left\langle\mathbf{V}^{\dagger}, \mathbf{V}\right\rangle=1$. Hence, from (4.7), apart from the two trivial eigenvalues (4.9), the rest of the eigenvalues satisfy

$$
\operatorname{Re}(\lambda) \sim-B \mathcal{E} / 2+\left\{\begin{array}{cl}
4 \delta C / D & k \text { odd }, \\
0 & k \text { even }(k \neq 0) .
\end{array}\right.
$$

By (4.21), the eigenvalues for $k$ even $(k \neq 0)$ are strictly stable. Consequently, the condition for stability is determined by the odd $k$ modes, i.e.

$$
-B \mathcal{E} / 2+4 \delta C / D<0 .
$$

The term $-B \mathcal{E} / 2$ in (4.21) can easily be understood as the result of the zeroth-order dissipative term in (3.11). The term $4 \delta C / D$ is a result of the discreteness in the problem. Solving for $\mathcal{E}$, we obtain the condition for stability

$$
\mathcal{E}>\frac{8 C}{B D} \delta
$$

and this reduces to (4.1) when the values (3.12) are substituted for $B, C$, and $D$.

\section{Calculation of the entire spectrum}

In this section, we calculate all of the eigenvalues in the spectrum for $\delta \rightarrow 0$. This will refine the results of the previous section and allows us to more accurately 
describe the structure of the set of eigenvalues. A conclusion of this analysis will be that the prediction of (4.1) is correct. In fact, for $k$ odd, the eigenvalues not well estimated by the perturbation analysis are more stable (i.e., have smaller, more negative real parts) than those captured accurately by the perturbation analysis; it is these accurately predicted eigenvalues that first cross the imaginary axis as $\mathcal{E}$ decreases.

The appearance of two cases in (4.21) is related to the existence of a symmetry in the eigenvalue problem (3.14): i.e., these equations are unchanged by the transformation

$$
\mathcal{T}:\left\{\begin{array}{cc}
W_{n} \mapsto-W_{N+1-n}, & n=1,2, \ldots, N, \\
P_{n} \mapsto P_{N-n}, & n=1,2, \ldots, N-1, \\
P_{0} \mapsto P_{0} &
\end{array}\right.
$$

that reflects the solutions around $x_{M}=M \delta$, where

$$
M=\frac{N+1}{2} .
$$

The point $x_{M}$ is the center of the interval outside the shear band. Let us define odd and even subspaces of $\mathbb{C}^{2 N}$,

$$
\begin{aligned}
& \mathcal{V}_{\mathrm{I}}=\left\{\mathbf{V} \in \mathbb{C}^{2 N}: \mathcal{T} \mathbf{V}=-\mathbf{V}\right\} \\
& \mathcal{V}_{\mathrm{II}}=\left\{\mathbf{V} \in \mathbb{C}^{2 N}: \mathcal{T} \mathbf{V}=\mathbf{V}\right\}
\end{aligned}
$$

We shall refer to an eigenvector $\mathbf{V}$, and its associated eigenvalue, as being of Type $I$ or Type II according to whether $\mathbf{V}$ belongs to $\mathcal{V}_{\mathrm{I}}$ or $\mathcal{V}_{\mathrm{II}}$, respectively. In (4.21), even and odd $k$ correspond to Type I and Type II eigenvalues, respectively.

The dimensions of $\mathcal{V}_{\mathrm{I}}$ and $\mathcal{V}_{\mathrm{II}}$ depend on whether $N=\delta^{-1}$ is odd or even. For definiteness we henceforth make the following

Assumption: $\quad N$ is even.

If $N$ were odd, the analysis would be different only in minor details.

We shall use asymptotic analysis to derive the full behavior of the eigenvalues analytically. In applying asymptotics, we shall consider the distinguished limit motivated by (4.1),

$$
\mathcal{E}=E \delta
$$

as $\delta \rightarrow 0$, where $E$ is a constant.

If (4.21) were uniformly valid in $k$, then substituting (5.4) into (4.15a, 4.21), we might expect (3.14) to have eigenvalues as follows:

Type I: $\quad \lambda=0$

$$
\lambda_{k} \approx \pm 2 k i \pi \sqrt{A E \delta}-(B E / 2) \delta, \quad k=1,2, \ldots, N / 2-1
$$

Type II: $\lambda \approx-D E$

$$
\begin{aligned}
\lambda_{k} \approx \pm & (2 k+1) i \pi \sqrt{A E \delta} \\
& +(-B E / 2+4 C / D) \delta,
\end{aligned}
$$


To understand the counting of Type I and Type II eigenvalues, suppose that a vector $\mathbf{V}$, with components $W_{n}, P_{n}$, belongs to $\mathcal{V}_{I}$. Since $\mathbf{V}$ is odd under (5.1), not all its components are independent; specifically,

$$
\begin{array}{cl}
W_{n}=W_{N+1-n}, & n=N / 2+1, N / 2+2, \ldots, N \\
P_{n}=-P_{N-n}, & n=N / 2, N / 2+1, \ldots, N \\
P_{0}=-P_{0} . &
\end{array}
$$

Hence, $P_{0}=0, P_{N / 2}=0$, and all components of $\mathbf{V}$ can be expressed in terms of

$$
W_{n}, n=1,2, \ldots, N / 2 \quad \text { and } \quad P_{n}, n=1,2, \ldots, N / 2-1,
$$

these $N-1$ components being arbitrary. Therefore, under assumption (5.3),

$$
\operatorname{dim} \mathcal{V}_{\mathrm{I}}=N-1 ; \quad \text { moreover } \quad \operatorname{dim} \mathcal{V}_{\mathrm{II}}=N+1,
$$

the second equation following either by counting subspace dimensions or by analysis similar to that for $\mathcal{V}_{\mathrm{I}}$.

For the Type I eigenvalues, the implication for stability of the prediction (5.5) is essentially correct: although $\operatorname{Im}\left(\lambda_{k}\right)$ differs significantly from (5.5) when $k$ is large, $\operatorname{Re}\left(\lambda_{k}\right)$ does not. Consequently, the Type I eigenmodes never cause instability. This conclusion is reached in Section 5.1.

By contrast, for the Type II eigenvalues, Re $\left(\lambda_{k}\right)$ depends on $k$ in a nontrivial way. As illustrated in the computation shown in Figure 6, the Type II eigenvalues lie along a curve in the complex plane, and the perturbation-theory estimates describe only the "nose" of this curve: i.e., the portion near the intersection of the curve with the real axis. To derive the complete behavior analytically, we shall need to let the enumeration parameter $k$ vary and to analyze several distinguished limits in $k, \delta$. While pursuing the complexities of this lengthy argument in Section 5.2, (5.5) will be a useful point of reference.

\section{$5.1 \quad$ Type I eigenvalues}

Since the entries of the matrix (3.14) do not depend on $n$, we may look for eigenvectors that have trigonometric dependence on $n$. Requiring that the eigenvector be odd under (5.1) gives the ansatz

$$
\begin{aligned}
W_{n} & =\cos [\kappa(n-M) \delta] \hat{W}, & & n=1,2, \ldots, N \\
P_{n} & =\sin [\kappa(n+1 / 2-M) \delta] \hat{P}, & & n=1,2, \ldots, N-1 \\
P_{0} & =0, & &
\end{aligned}
$$

where $\hat{W}$ and $\hat{P}$ are constants. By analogy with continuous problems, we shall call $\kappa$ the wavenumber; this parameter cannot be determined independently of $\lambda$. 
Substituting (5.7) into the eigenvalue problem (3.14) yields the system of equations,

$$
\begin{aligned}
\lambda \hat{W} & =2 \delta^{-1} \sin (\kappa \delta / 2) \hat{P} \\
\mathcal{E}^{-1} \lambda \hat{P} & =-2 A \delta^{-1} \sin (\kappa \delta / 2) \hat{W}-B \hat{P} \\
\lambda \cos (\kappa[1-\delta] / 2) \hat{W} & =-\delta^{-1} \sin (\kappa[1-2 \delta] / 2) \hat{P}
\end{aligned}
$$

Here, (5.8a) assures that (3.14a) is satisfied for all $n$ except $n=N$. Likewise, (5.8b) assures that (3.14b) is satisfied for all $n$ (except $n=0$ ). Equation (3.14a) for $n=N$ leads to $(5.8 \mathrm{c})$ when, using $(3.13), P_{N}$ is replaced by $P_{0}$, and it is recalled from (5.7c) that $P_{0}=0$. Equation (3.14c) is satisfied without imposing any restriction on $\hat{W}, \hat{P}$ : to see this, replace $W_{0}$ by $W_{N}$ in $(3.14 \mathrm{c})$ and observe from (5.1) that for a Type I eigenvector, $P_{0}=0$ and $W_{N}=W_{1}$.

Equations (5.8) are a system of three homogeneous linear equations for the two unknowns $\hat{W}, \hat{P}$ that can be written in matrix form as

$$
\left(\begin{array}{cc}
\lambda & -2 \delta^{-1} \sin (\kappa \delta / 2) \\
2 A \delta^{-1} \sin (\kappa \delta / 2) & \mathcal{E}^{-1} \lambda+B \\
\lambda \cos (\kappa[1-\delta] / 2) & \delta^{-1} \sin (\kappa[1-2 \delta] / 2)
\end{array}\right)\left(\begin{array}{c}
\hat{W} \\
\hat{P}
\end{array}\right)=\left(\begin{array}{l}
0 \\
0 \\
0
\end{array}\right)
$$

In order for this system to have a nontrivial solution, each $2 \times 2$ block of the coefficient matrix must be singular. Setting the determinants of rows 1 and 2 and of rows 1 and 3 equal to zero, we obtain a system of two equations for $\lambda$ and $\kappa$,

$$
\begin{gathered}
\mathcal{E}^{-1} \lambda^{2}+B \lambda+4 A \delta^{-2} \sin ^{2}(\kappa \delta / 2)=0 . \\
\lambda \sin (\kappa / 2)=0 .
\end{gathered}
$$

In deriving (5.10b), we have used the trigonometric identity $\sin (\phi-2 \psi)+2 \cos (\phi-$ $\psi) \sin \psi=\sin \phi$ with $\phi=\kappa / 2$ and $\psi=\kappa \delta / 2$.

From (5.10b), we observe that either $\lambda=0$ or $\sin (\kappa / 2)=0$. Regarding nonzero eigenvalues, we deduce that

$$
\kappa=2 \pi k, \quad k=1,2, \ldots, N / 2-1 .
$$

We claim that extending the range of $k$ in (5.11) would not lead to additional eigenvalues. Arguing indirectly, this claim follows from counting: for each of the $N / 2-1$ values of $k$ listed in (5.11), there are two solutions of (5.10a),

$$
\lambda=\frac{\mathcal{E}}{2}\left\{-B \pm \sqrt{B^{2}-16 \delta^{-2} A \mathcal{E}^{-1} \sin ^{2}(k \pi \delta)}\right\},
$$

and all of these eigenvalues are distinct. Thus, the eigenvalues enumerated by (5.11), together with the zero eigenvalue, account for all of the $N-1$ possible eigenvectors in $\mathcal{V}_{I}$.

It is instructive also to prove the claim directly. First we argue that neither of the transformations
(a) $\kappa \mapsto-\kappa$
(b) $\kappa \mapsto \kappa+2 \pi N$, 
operating on (5.7), leads to a new eigenvector. Under (5.13a), the cosine factor in (5.7a) is unchanged and the sine factor in $(5.7 \mathrm{~b})$ reverses its sign; since the latter may be absorbed into a redefinition of $\hat{P}$, no new eigenvector results. Under (5.13b),

$$
\cos [(\kappa+2 \pi N)(n-M) \delta]=\cos [\kappa(n-M) \delta+2 \pi(n-M)]=-\cos [\kappa(n-M) \delta]
$$

since $n-M$ is a half integer so $2 \pi(n-M)=\pi$, modulo $2 \pi$; similarly,

$$
\sin [(\kappa+2 \pi N)(n+1 / 2-M) \delta]=\sin [\kappa(n+1 / 2-M) \delta]
$$

since $n+1 / 2-M$ is an integer; again no new eigenvector results. Using (5.11) to relate $\kappa$ to $k$, we rewrite the transformations (5.13) as

$$
\text { (a) } k \mapsto-k \quad \text { (b) } k \mapsto k+N \text {. }
$$

Since any integer can be obtained from $\{0,1, \ldots, N / 2\}$ by repeated applications of (5.14), it suffices to consider just $k=0,1, \ldots, N / 2$ in (5.11). If $k=0$, then from (5.7), $\quad W_{n}=1, P_{n}=0$, and by (5.10a), $\lambda=0$ : i.e., $k=0$ repeats the zero eigenvalue obtained above . The situation with $k=N / 2$ is the same as $k=0$ : this value of $k$ merely reproduces the zero eigenvalue in the same way. Therefore, all nonzero eigenvalues are accounted for by the enumeration in (5.11).

Note from (5.12) that, no matter what the size of $\mathcal{E}$ and $\delta$ are, all nonzero Type I eigenvalues lie in the stable half plane. In the distinguished limit $\mathcal{E}=E \delta$ as $\delta \rightarrow 0$, the radical in (5.12) is pure imaginary, and each eigenvalue in (5.12) is asymptotic to the corresponding eigenvalue in (5.5), modulo an $O\left(\delta^{3 / 2}\right)$ error. However, this convergence is not uniform in $k$.

In terms of the continuum approximation of Section 4.1, the $P$-component of the eigenvector (5.7) corresponds to the function

$$
P(x)=\hat{P} \sin [\kappa(x-1 / 2)],
$$

modulo an $O(\delta)$ translation. Equation (5.11) guarantees that the boundary conditions (4.14) in the continuous problem are satisfied. While the perturbation-theory argument of Section 4.1 shows only that (4.14) is satisfied to leading order, the calculation here is exact.

\subsection{Type-II eigenvalues}

\section{(a) Preliminaries}

\footnotetext{
I When $k=0$, one might wonder why the other root of (5.10a), $\lambda=-B \mathcal{E}$, is not an eigenvalue. The reason is that, although (5.9) has a solution $\hat{W}, \hat{P}$, in which $\hat{W}=0$ and $\hat{P} \neq 0$, nonetheless, on substitution into equations (5.7), relating eigenvectors to $\hat{W}, \hat{P}$, we obtain that $W_{n}=0$ and $P_{n}=0$ for all $n$, since $k=\kappa=0$. Consequently, this solution of (5.9) does not correspond to an eigenvector.
} 
For Type II eigenvalues, we seek eigenvectors that are even under the transformation (5.1). The appropriate ansatz, analogous to (5.7), is

$$
\begin{array}{rlrl}
W_{n} & =\sin [\kappa(n-M) \delta] \hat{W}, & n=1,2, \ldots, N \\
P_{n} & =\cos [\kappa(n+1 / 2-M) \delta] \hat{P}, & & n=1,2, \ldots, N-1 \\
P_{0} & =\hat{S} . &
\end{array}
$$

In the continuum approximation, the $P$-component of the eigenvector (5.15) corresponds to the function $P(x)=\hat{P} \cos [\kappa(x-1 / 2)]$. For $P(x)$ to satisfy (4.14), the wave number $\kappa$ must satisfy

$$
\cos (\kappa / 2)=0,
$$

which has solutions $\kappa=(2 k+1) \pi$. To determine the appropriate range for $k$, we argue as for the Type I eigenvalues: (i) neither of the transformations (5.13) leads to a new eigenvector, and (ii) any complex number $\kappa$ can be obtained from one in

$$
\{\kappa: 0 \leq \operatorname{Re} \kappa \leq \pi N\}
$$

by repeated application of (5.13). Therefore it suffices to consider wave numbers in this set. The solutions of (5.16) in the strip (5.17) are

$$
\kappa=(2 k+1) \pi, \quad k=0,1, \ldots, N / 2-1 .
$$

One might conjecture that (5.18) gives the condition for (5.15) to be an eigenvector. As we shall show below, apart from the real eigenvalue $\lambda \approx-D E$, to leading order this conjecture is correct. However, the higher-order corrections to $\kappa$, which are complex-valued, greatly complicate the proof.

If (5.15) is substituted into the equations (3.14), the system of $2 N$ equations collapses into a homogeneous system of four equations in three unknowns:

$$
\begin{aligned}
\lambda \hat{W} & =-2 \delta^{-1} \sin (\kappa \delta / 2) \hat{P} \\
\mathcal{E}^{-1} \lambda \hat{P} & =2 A \delta^{-1} \sin (\kappa \delta / 2) \hat{W}-B \hat{P} \\
\mathcal{E}^{-1} \lambda \hat{S} & =2 C \sin (\kappa[1-\delta] / 2) \hat{W}-D \delta^{-1} \hat{S} \\
\lambda \sin (\kappa[1-\delta] / 2) \hat{W} & =\delta^{-1}(\hat{S}-\cos (\kappa[1-2 \delta] / 2) \hat{P}) .
\end{aligned}
$$

Similar to the derivation of (5.8), equation (5.19a) assures that (3.14a) is satisfied for all $n$ except $n=N,(5.19 \mathrm{~b})$ assures that (3.14b) is satisfied for all $n,(3.14 \mathrm{c})$ leads to (5.19c), and (3.14a) with $n=N$ leads to $(5.19 \mathrm{~d})$. We use (5.19d) to eliminate $\hat{S}$ and rewrite the remaining equations in matrix form:

$$
\left(\begin{array}{cc}
\lambda & 2 \delta^{-1} \sin (\kappa \delta / 2) \\
-2 A \delta^{-1} \sin (\kappa \delta / 2) & \mathcal{E}^{-1} \lambda+B \\
\left(\mathcal{E}^{-1} \delta \lambda^{2}+D \lambda-2 C\right) \sin (\kappa[1-\delta] / 2) & \left(\mathcal{E}^{-1} \lambda+\delta^{-1} D\right) \cos (\kappa[1-2 \delta] / 2)
\end{array}\right)\left(\begin{array}{c}
\hat{W} \\
\hat{P}
\end{array}\right)=\left(\begin{array}{l}
0 \\
0 \\
0
\end{array}\right) .
$$


For a nonzero solution $\hat{W}, \hat{P}$ of (5.20) to exist, each $2 \times 2$ block of the coefficient matrix must be singular. After replacing $\mathcal{E}$ by $E \delta$ and simplifying, we express this condition as the system of two equations for $\lambda$ and $\kappa$

$$
\begin{gathered}
E^{-1} \lambda^{2}+B \delta \lambda+4 A \delta^{-1} \sin ^{2}(\kappa \delta / 2)=0 \\
\left(E^{-1} \lambda^{2}+D \lambda\right) \cos (\kappa / 2)+4 C f(\kappa, \delta)=0
\end{gathered}
$$

where

$$
f(\kappa, \delta)=\sin (\kappa \delta / 2) \cos (\kappa \delta / 2) \sin (\kappa / 2)-\sin ^{2}(\kappa \delta / 2) \cos (\kappa / 2)
$$

is introduced only to make more visible the structure of the equations: i.e., two quadratic equations in $\lambda$.

\section{(b) Elimination of $\lambda$ using the resolvent}

In general, for a pair quadratic equations,

$$
\begin{aligned}
a_{2} \lambda^{2}+a_{1} \lambda+a_{0} & =0 \\
b_{2} \lambda^{2}+b_{1} \lambda+b_{0} & =0
\end{aligned}
$$

to have a common solution, their resultant must be zero [14]: i.e.,

$$
\left(2 a_{2} b_{0}-a_{1} b_{1}+2 a_{0} b_{2}\right)^{2}-\left(4 a_{2} a_{0}-a_{1}^{2}\right)\left(4 b_{2} b_{0}-b_{1}^{2}\right)=0 .
$$

Substituting the coefficients in (5.21) into (5.23), we derive a transcendental equation for $\kappa$ :

$$
\mathbf{a}(\kappa, \delta) \cos ^{2}(\kappa / 2)+\mathbf{b}(\kappa, \delta) \sin (\kappa / 2) \cos (\kappa / 2)+\mathbf{c}(\kappa, \delta) \sin ^{2}(\kappa / 2)=0,
$$

where the coefficients are given by

$$
\begin{aligned}
& \mathbf{a}(\kappa, \delta)=\left[4 A^{2}+O(\delta)\right] \sigma^{3}+\left[A D^{2} E \delta+O\left(\delta^{2}\right)\right] \sigma \\
& \mathbf{b}(\kappa, \delta)=\left\{\left[-8 A C \delta+O\left(\delta^{2}\right)\right] \sigma^{2}-\left[B C D E \delta^{3}+O\left(\delta^{4}\right)\right]\right\} \sqrt{1-\sigma^{2}} \\
& \mathbf{c}(\kappa, \delta)=\left[4 C^{2} \delta^{2}\right] \sigma\left(1-\sigma^{2}\right),
\end{aligned}
$$

with

$$
\sigma=\sigma(\kappa, \delta)=\sin (\kappa \delta / 2) .
$$

Note that the positive square root is taken in (5.25), since $\cos (\kappa \delta / 2)=\sqrt{1-\sigma^{2}}$ is positive, in the range (5.18).

A common factor of $\sin (\kappa \delta / 2) / E^{2} \delta^{2}$ has been factored out and removed from all coefficients in (5.25). The full expressions for these coefficients are given in Table 1. Since the non-constant factor $\sin (\kappa \delta / 2)$ vanishes at a point in $(5.17)-$ i.e., $\kappa=0$ one might wonder whether any eigenvalues of (3.14) are lost through dividing by zero. In fact, no eigenvalues are lost because, although a nonzero solution $\hat{W}, \hat{P}$ of (5.20) does exist for this value of $\kappa$, the solution has $\hat{P}=0$, so that the proposed eigenvector (5.15) vanishes identically. 


$$
\begin{aligned}
\frac{\mathbf{a}}{E^{2} \delta^{2}}= & \left(4 A^{2} E^{-2} \delta^{-2}+8 A C E^{-2} \delta^{-1}+4 C^{2} E^{-2}\right) \sigma^{3}+ \\
& \left(A D^{2} E^{-1} \delta^{-1}-A B D E^{-1}+B C D E^{-1} \delta-B^{2} C E^{-1} \delta^{2}\right) \sigma \\
\frac{\mathbf{b}}{E^{2} \delta^{2}=} & \left(-8 A C E^{-2} \delta^{-1}-8 C^{2} E^{-2}\right) \sigma^{2} \sqrt{1-\sigma^{2}}+ \\
& \left(-B C D E^{-1} \delta+B^{2} C E^{-1} \delta^{2}\right) \sqrt{1-\sigma^{2}} \\
\frac{\mathbf{c}}{E^{2} \delta^{2}=} & 4 C^{2} E^{-2} \sigma\left(1-\sigma^{2}\right)
\end{aligned}
$$

Table 1: Full expansion of the coefficients in (5.25), in which $\sigma=\sin (\kappa \delta / 2)$.

Because of the connection of solutions of (5.24) with Type II eigenvalues of (3.14), we need to find only $N+1$ solutions of (5.24) that lead to distinct eigenvalues. Our analysis of (5.24) is based on showing that as $\delta \rightarrow 0$ the equation reduces to the leading order problem $\mathbf{a}(\kappa, \delta) \cos ^{2}(\kappa / 2)=0$. All but one of the solutions of (5.24) are complex conjugate pairs that come from perturbations of (real) zeros of $\cos ^{2}(\kappa / 2)$. As we now show, however, one solution comes from a zero of $\mathbf{a}(\kappa, \delta)$ along the imaginary axis; this solution is associated with the real eigenvalue in (5.5), $\lambda \approx-D E$.

\section{(c) The real eigenvalue}

Lemma 5.1 Equation (5.24) has a solution with the asymptotic form

$$
\kappa=i \frac{D \sqrt{E}}{\sqrt{A}} \delta^{-\frac{1}{2}}+O(1) \quad \text { as } \delta \rightarrow 0 .
$$

Proof: Let us substitute $\kappa=i \tilde{\kappa} \delta^{-1 / 2}$ into equation (5.24), divide by $i \cosh ^{2}\left(\tilde{\kappa} \delta^{-1 / 2} / 2\right)$, and rewrite the resulting equation in the form

$$
\tilde{\mathbf{a}}(\tilde{\kappa}, \delta)+\tilde{\mathbf{b}}(\tilde{\kappa}, \delta) \tanh \left(\tilde{\kappa} \delta^{-1 / 2} / 2\right)+\tilde{\mathbf{c}}(\tilde{\kappa}, \delta) \tanh ^{2}\left(\tilde{\kappa} \delta^{-1 / 2} / 2\right)=0 .
$$

Of course as $\delta \rightarrow 0$, we have $\tanh \left(\tilde{\kappa} \delta^{-1 / 2} / 2\right) \sim 1$, modulo an exponentially small error. Since

$$
\begin{aligned}
& \sin (\kappa \delta / 2)=i \sinh \left(\tilde{\kappa} \delta^{1 / 2} / 2\right)=(i \tilde{\kappa} / 2) \delta^{1 / 2}+O\left(\delta^{3 / 2}\right), \\
& \cos (\kappa \delta / 2)=\cosh \left(\tilde{\kappa} \delta^{1 / 2} / 2\right)=1+O(\delta),
\end{aligned}
$$

we see from (5.25) that

$$
\begin{aligned}
\tilde{\mathbf{a}}(\tilde{\kappa}, \delta) & =\frac{1}{2}\left(A D^{2} E-A^{2} \tilde{\kappa}^{2}\right) \delta^{3 / 2}+O\left(\delta^{5 / 2}\right), \\
\tilde{\mathbf{b}}(\tilde{\kappa}, \delta) & =O\left(\delta^{2}\right), \\
\tilde{\mathbf{c}}(\tilde{\kappa}, \delta) & =O\left(\delta^{5 / 2}\right) .
\end{aligned}
$$

Note that the leading-order term in $\tilde{\mathbf{a}}(\tilde{\kappa}, \delta)$ vanishes if $\tilde{\kappa}=D \sqrt{E} / \sqrt{A}$. Moreover, the modified coefficients $\tilde{\mathbf{a}}, \tilde{\mathbf{b}}, \tilde{\mathbf{c}}$ are real if $\tilde{\kappa}$ is real. Synthesizing the above information, we conclude that the leading-order zero of (5.28) persists under perturbation, 
provided $\delta$ is small enough.

Given the estimate (5.27) for $\kappa$, it is easily verified that $\lambda=-D E+O(\sqrt{\delta})$ is the common solution of $(5.21 \mathrm{a}, \mathrm{b})$ and hence an eigenvalue of (3.14). This repeats the value found in (5.5).

It is apparent from the above proof that (5.24) also has a solution $\kappa \approx-i D \sqrt{E} / \sqrt{A}$. However, this second solution gives rise to the same eigenvalue since the transformation $\kappa \mapsto-\kappa$ does not generate a new eigenvector.

(d) The leading-order solution of (5.24) for the (approximately) real wave-numbers

Proposition 5.2 For each $k=0,1, \ldots, N / 2$ - 1, equation (5.24) has two complex conjugate solutions with the asymptotic form

$$
\kappa=(2 k+1) \pi+O(\sqrt{\delta}) \quad \text { as } \delta \rightarrow 0 .
$$

Moreover, the error estimate is uniform in $k$ : i.e., there are positive constants $\delta_{*}$ and $\Gamma$ such that for all $\delta \in\left(0, \delta_{*}\right)$ and for $k=0,1, \ldots, N / 2-1$ the corresponding solutions satisfy

$$
|\kappa-(2 k+1) \pi| \leq \Gamma \sqrt{\delta} .
$$

Remarks: (i) The eigenvalue $\lambda \approx-D E$ and the $N$ eigenvalues derived from (5.29) account for all the Type-II eigenvalues. (ii) The $O(\sqrt{\delta})$-correction in (5.29) represents the start of an asymptotic series in $\sqrt{\delta}$, which we will develop below.

To prove the proposition, we examine equation (5.24) for $\kappa$ in the union of small neighborhoods of the leading order terms in (5.29):

$$
\Omega=\bigcup_{k=0}^{N / 2-1}\{\kappa:|\kappa-(2 k+1) \pi| \leq 1\} .
$$

Lemma 5.3 There are positive constants $\delta_{*}$ and $\Gamma$ such that, for all $\kappa \in \Omega$ and all $\delta<\delta_{*}$, the function $\sigma=\sin (\kappa \delta / 2)$ satisfies

$$
\text { (a) } \operatorname{Re}\left(\sigma^{2}\right) \geq 0 \quad \text { and } \quad \text { (b) } \Gamma^{-1} \delta \leq|\sigma| \leq 2 \text {. }
$$

Proof: We claim that for $\kappa \in \Omega$

$$
\delta<\operatorname{Re}(\kappa \delta / 2)<\pi / 2, \quad|\operatorname{Im}(\kappa \delta / 2)| \leq \delta / 2 .
$$

The bound on $\operatorname{Im}(\kappa \delta / 2)$ is trivial. The smallest value for $\operatorname{Re}(\kappa \delta / 2)$ in $\Omega$, which is achieved on the "first" disk $\{\kappa:|\kappa-\pi| \leq 1\}$, is $(\pi-1) \delta / 2$, from which the lower bound in (5.32) follows. The largest value for $\operatorname{Re}(\kappa \delta / 2)$ in $\Omega$, which is achieved on the "last" disk $\{\kappa:|\kappa-(N-1) \pi| \leq 1\}$, is $[(N-1) \pi+1] \delta / 2$; the claim follows on recalling that $N=\delta^{-1}$. 
To verify (5.31a), observe that $\operatorname{Re}\left(\sigma^{2}\right) \geq 0$ if and only if $|\operatorname{Im} \sigma| \leq|\operatorname{Re} \sigma|$, or $|\operatorname{Im}(\sigma) / \operatorname{Re}(\sigma)| \leq 1$. Recalling that $\sin (x+i y)=\sin x \cosh y+i \cos x \sinh y$, we see that

$$
\left|\frac{\operatorname{Im}(\sigma)}{\operatorname{Re}(\sigma)}\right|=\left|\frac{\tanh (\operatorname{Im}(\kappa \delta / 2))}{\tan (\operatorname{Re}(\kappa \delta / 2))}\right| .
$$

Since $|\tanh y| \leq|y|$ and, provided $|x|<\pi / 2, \quad|\tan x| \geq|x|$, we conclude that

$$
\left|\frac{\operatorname{Im}(\sigma)}{\operatorname{Re}(\sigma)}\right| \leq\left|\frac{\operatorname{Im}(\kappa \delta / 2)}{\operatorname{Re}(\kappa \delta / 2)}\right|=\left|\frac{\operatorname{Im}(\kappa)}{\operatorname{Re}(\kappa)}\right| .
$$

The inequality (5.31a) follows on remarking that $|\operatorname{Im}(\kappa) / \operatorname{Re}(\kappa)| \leq 1$ in $\Omega$.

To verify the lower bound in $(5.31 \mathrm{~b})$, observe that $(\sin z) / z$ is analytic and nonvanishing in the closed rectangle

$$
\mathcal{R}=\{z: 0 \leq \operatorname{Re}(z) \leq \pi / 2,|\operatorname{Im}(z)| \leq 1 / 2\} .
$$

Therefore there exists a positive constant $\Gamma$ such that, for $z \in \mathcal{R}, \quad|\sin z| \geq \Gamma^{-1}|z|$. By (5.32), provided $\delta<1, \kappa \delta / 2$ belongs to $\mathcal{R}$. Therefore,

$$
|\sigma| \geq \Gamma^{-1}|\kappa \delta / 2| \geq \Gamma^{-1} \operatorname{Re}(\kappa \delta / 2) \geq \Gamma^{-1} \delta,
$$

where we have invoked (5.32) for the last inequality.

To verify the upper bound in (5.31b), observe that since $|\operatorname{Im}(\kappa \delta / 2)| \leq \delta / 2$

$$
\sin (\kappa \delta / 2)=\sin (\operatorname{Re}(\kappa \delta / 2))+O(\delta) .
$$

Of course $\sin (\operatorname{Re}(\kappa \delta / 2)) \leq 1$, so if $\delta$ is sufficiently small, we have $|\sigma| \leq 2$.

Lemma 5.4 If $z \in \mathbb{C}$ and $c \in \mathbb{R}$ satisfy $\operatorname{Re}(z) \geq 0$ and $c \geq 0$, then

$$
|z+c| \geq(|z|+c) / \sqrt{2}
$$

Proof: For the right-hand side of this inequality, we have

$$
(|z|+c)^{2}=|z|^{2}+2|z| c+c^{2} \leq 2\left(|z|^{2}+c^{2}\right),
$$

and for the left-hand side,

$$
|z+c|^{2}=|z|^{2}+2 \operatorname{Re}(z) c+c^{2} \geq|z|^{2}+c^{2} .
$$

The result follows on combining these.

Lemma 5.5 There are positive constants $\delta_{*}$ and $\Gamma$ such that, if $\kappa \in \Omega$ and $\delta<\delta_{*}$, then $\mathbf{a}(\kappa, \delta)$ is nonzero and in fact satisfies

$$
|\mathbf{a}(\kappa, \delta)| \geq \Gamma^{-1}\left(|\sigma|^{2}+\delta\right)|\sigma| .
$$


Proof: The leading order coefficients in (5.25a) - i.e., $4 A^{2}$ and $A D^{2} E \delta$ - are both positive. Therefore, if $\delta$ is sufficiently small, $\mathbf{a}(\kappa, \delta)=\left(\gamma_{1} \sigma^{2}+\gamma_{2} \delta\right) \sigma$, where $\gamma_{1}$ and $\gamma_{2}$ are positive and may be bounded from below, say

$$
\left|\gamma_{i}\right| \geq \sqrt{2} \Gamma^{-1}
$$

By Lemma 5.3, $\operatorname{Re}\left(\sigma^{2}\right) \geq 0$. We obtain the result on substituting $z=\gamma_{1} \sigma^{2}$ and $c=\gamma_{2} \delta$ into Lemma 5.4 and invoking (5.33).

Lemma 5.6 There are positive constants $\delta_{*}$ and $\Gamma$ such that, for all $\kappa \in \Omega$ and all $\delta<\delta_{*}$,

$$
\text { (a) }\left|\frac{\mathbf{b}(\kappa, \delta)}{\mathbf{a}(\kappa, \delta)} \sin (\kappa / 2)\right| \leq \Gamma \sqrt{\delta} \quad \text { and } \quad \text { (b) }\left|\frac{\mathbf{c}(\kappa, \delta)}{\mathbf{a}(\kappa, \delta)} \sin ^{2}(\kappa / 2)\right| \leq \Gamma \delta .
$$

Proof. Since the proof of (5.34b) is rather easy, we focus on (5.34a). The factor $\sin (\kappa / 2)$ admits the easy bound $|\sin (\kappa / 2)| \leq \cosh 1 / 2$. It follows from $(5.25 \mathrm{~b})$ that

$$
|\mathbf{b}(\kappa, \delta)| \leq \Gamma\left(|\sigma|^{2}+\delta^{2}\right) \delta .
$$

Combining this estimate with Lemma 5.5, we conclude that

$$
\left|\frac{\mathbf{b}(\kappa, \delta)}{\mathbf{a}(\kappa, \delta)} \sin (\kappa / 2)\right| \leq \Gamma \frac{\left(|\sigma|^{2}+\delta^{2}\right) \delta}{\left(|\sigma|^{2}+\delta\right)|\sigma|}
$$

for an appropriately increased constant $\Gamma$.

Let us denote the right-hand side of (5.35) by $\phi(|\sigma|, \delta)$. By Lemma 5.3, for $\kappa \in \Omega$, we have $\Gamma^{-1} \delta \leq|\sigma| \leq 2$. At the lower and upper extremes for $|\sigma|$,

$$
\phi\left(\Gamma^{-1} \delta, \delta\right)=O(\delta) \quad \text { and } \quad \phi(2, \delta)=O(\delta),
$$

respectively. Applying calculus arguments, we find that $\phi(|\sigma|, \delta)$ has an interior maximum at a point for which $|\sigma| \sim \gamma \delta^{1 / 2}$, and here

$$
\phi\left(\gamma \delta^{1 / 2}, \delta\right)=O\left(\delta^{1 / 2}\right) .
$$

This proves Lemma 5.6.

Proof (of Proposition 5.2): According to Lemma 5.5, a $(\kappa, \delta)$ does not vanish for $\kappa \in \Omega$. Therefore we may divide $(5.24)$ by $\mathbf{a}(\kappa, \delta)$ to obtain

$$
\cos ^{2}(\kappa / 2)+\frac{\mathbf{b}}{\mathbf{a}} \sin (\kappa / 2) \cos (\kappa / 2)+\frac{\mathbf{c}}{\mathbf{a}} \sin ^{2}(\kappa / 2)=0 .
$$

The leading-order version of $(5.36), \cos ^{2}(\kappa / 2)=0$, has double roots at $\kappa=(2 k+$ 1) $\pi$ for $k=0,1, \ldots, N-1$. By complex function theory, a perturbed equation $\cos ^{2}(\kappa / 2)+f(\kappa)=0$ has exactly two roots in the disk $\{\kappa:|\kappa-(2 k+1) \pi| \leq 1\}$ provided the perturbing function $f$ is sufficiently small. According to Lemma 5.6, 
we can make the perturbation arbitrarily small by taking $\delta$ small. This establishes the existence of the two roots.

To prove (5.30), we regard (5.36) as a quadratic equation in $\cos (\kappa / 2)$. Substituting the estimate of Lemma 5.6 into

$$
\cos (\kappa / 2)=\left[-\frac{\mathbf{b}}{2 \mathbf{a}} \pm \sqrt{\left(\frac{\mathbf{b}}{2 \mathbf{a}}\right)^{2}-\frac{\mathbf{c}}{\mathbf{a}}}\right] \sin (\kappa / 2),
$$

we conclude that $\cos (\kappa / 2)=O(\sqrt{\delta})$, from which (5.30) follows.

\section{(e) Asymptotic series for the eigenvalues}

In this subsection we find asymptotic series by analyzing equations (5.21) directly; for convenience, we reproduce these equations here:

$$
\begin{gathered}
E^{-1} \lambda^{2}+B \delta \lambda+4 A \delta^{-1} \sin ^{2}(\kappa \delta / 2)=0, \\
\left\{E^{-1} \lambda^{2}+D \lambda-4 C \sin ^{2}(\kappa \delta / 2)\right\} \cos (\kappa / 2) \\
+4 C \sin (\kappa \delta / 2) \cos (\kappa \delta / 2) \sin (\kappa / 2)=0 .
\end{gathered}
$$

One approach to the asymptotics as $\delta \rightarrow 0$ would be to develop an expansion for $\kappa$ from (5.24) and then substitute into (5.37) to derive a series for $\lambda$. However, having found the leading-order term for $\kappa$, it is easier to work with (5.37) exclusively, using these equations to find higher-order terms for $\kappa$ and the complete series for $\lambda$ simultaneously. We will follow the latter approach.

The range of $k$ in (5.29) is proportional to $\delta^{-1}$. To capture the full behavior of the eigenvalues over that large range, we will consider three distinguished limits as $\delta \rightarrow 0$ :
(a) $k$ fixed as $\delta \rightarrow 0$
(low wave numbers)
(b) $k \delta^{-1 / 2}$ fixed as $\delta \rightarrow 0 \quad$ (intermediate wave numbers)
(c) $k \delta^{-1}$ fixed as $\delta \rightarrow 0 \quad$ (high wave numbers).

The fact that $k$ is an integer makes limits (b) and (c) seem problematic. To explore this issue, let us examine (5.37) bearing in mind that $k$ is related to $\kappa$ through (5.29). Equations (5.37) depends on $\kappa$ through a "fast" variable - e.g., as in $\sin (\kappa / 2)$ - and through a "slow" variable - e.g., as in $\sin (\kappa \delta / 2)$. Although $\sin (\kappa \delta / 2)$ varies only slightly between two consecutive values of $\kappa$ in (5.29), the fast-variable function $\sin (\kappa / 2)$ varies over half a period for the same range of $\kappa$. In other words, the nature of (5.37) changes completely between consecutive values of $\kappa$ in (5.29).

To circumvent this difficulty, we rewrite (5.37) slightly. To leading order, (5.29) enumerates zeros of $\cos (\kappa / 2)$. Consequently, $\sin (\kappa / 2)$ will be nonzero at these values of $\kappa$, even allowing for the correction term. If we divide (5.37b) by $\sin (\kappa / 2)$, we will obtain an equation that depends on the fast variable only through $\cot (\kappa / 2)$. 
On an intuitive level, the key observation is that if $\kappa$ equals $(2 k+1) \pi$ plus a correction term, say $\kappa=(2 k+1) \pi+\tilde{\kappa}$, then

$$
\cot (\kappa / 2)=-\tan (\tilde{\kappa} / 2) .
$$

In other words, after the modification of (5.37), the dependence on the fast variable simplifies to dependence on the correction term, which by Proposition 5.2 is $O(\sqrt{\delta})$.

To formalize these ideas, we define $\theta=(2 k+1) \pi$, including a scale factor while interpolating $k$ to non-integer values; we set $\kappa=\theta+\tilde{\kappa}$; and we substitute into the modified (5.37) to obtain

$$
\begin{gathered}
E^{-1} \lambda^{2}+B \delta \lambda+4 A \delta^{-1} \sin ^{2}[(\theta+\tilde{\kappa}) \delta / 2]=0, \\
\left\{E^{-1} \lambda^{2}+D \lambda-4 C \sin ^{2}[(\theta+\tilde{\kappa}) \delta / 2]\right\} \tan (\tilde{\kappa} / 2) \\
-4 C \sin [(\theta+\tilde{\kappa}) \delta / 2] \cos [(\theta+\tilde{\kappa}) \delta / 2]=0 .
\end{gathered}
$$

In (5.37), for each $\delta$, the roots of two equations in $\lambda$ and $\kappa$ are to be determined. By contrast, for each $\delta,(5.38)$ defines $\lambda$ and $\tilde{\kappa}$ as functions of $\theta$, and the analogue of (5.29) is given by

$$
\kappa=\theta+\tilde{\kappa}(\theta, \delta), \quad \theta=\pi, 3 \pi, 5 \pi, \ldots,(N-1) \pi .
$$

Moreover, the proof of Proposition 5.2 may be extended to show that if $\delta<\delta_{*}$

$$
|\tilde{\kappa}(\theta, \delta)|<\Gamma \sqrt{\delta}, \quad \pi \leq \theta \leq N \pi .
$$

We turn now to the asymptotic solution of (5.38). It follows from (5.40) that in each of the three distinguished limits, the leading-order term in the expansion of $\tilde{\kappa}(\theta, \delta)$ is $O\left(\delta^{1 / 2}\right)$. By contrast, the series for $\lambda(\theta, \delta)$ begin with different powers of $\delta$ in the three cases. In Case (a) we obtain series in powers of $\delta^{1 / 2}$ as follows:

$$
\begin{aligned}
\tilde{\kappa}(\theta, \delta) & \sim \psi_{1}^{(a)}(\theta) \delta^{1 / 2}+\psi_{2}^{(a)}(\theta) \delta+\psi_{3}^{(a)}(\theta) \delta^{3 / 2}+\ldots \\
\lambda(\theta, \delta) & \sim \lambda_{1}^{(a)}(\theta) \delta^{1 / 2}+\lambda_{2}^{(a)}(\theta) \delta+\lambda_{3}^{(a)}(\theta) \delta^{3 / 2}+\ldots
\end{aligned}
$$

In Case (b), we let $\theta=\delta^{-1 / 2} \theta_{b}$, and we get series

$$
\begin{aligned}
\tilde{\kappa}\left(\delta^{-1 / 2} \theta_{b}, \delta\right) & \sim \kappa_{1}^{(b)}\left(\theta_{b}\right) \delta^{1 / 2}+\kappa_{2}^{(b)}\left(\theta_{b}\right) \delta+\kappa_{3}^{(b)}\left(\theta_{b}\right) \delta^{3 / 2}+\ldots \\
\lambda\left(\delta^{-1 / 2} \theta_{b}, \delta\right) & \sim \lambda_{0}^{(b)}\left(\theta_{b}\right)+\lambda_{1}^{(b)}\left(\theta_{b}\right) \delta^{1 / 2}+\lambda_{2}^{(b)}\left(\theta_{b}\right) \delta+\ldots
\end{aligned}
$$

In Case (c), we let $\theta=\delta^{-1} \theta_{c}$, and we get series

$$
\begin{aligned}
& \tilde{\kappa}\left(\delta^{-1} \theta_{c}, \delta\right) \sim \kappa_{1}^{(c)}\left(\theta_{c}\right) \delta^{1 / 2}+\kappa_{2}^{(c)}\left(\theta_{c}\right) \delta+\kappa_{3}^{(c)}\left(\theta_{c}\right) \delta^{3 / 2}+\ldots \\
& \lambda\left(\delta^{-1} \theta_{c}, \delta\right) \sim \lambda_{-1}^{(c)}\left(\theta_{c}\right) \delta^{-1 / 2}+\lambda_{0}^{(c)}\left(\theta_{c}\right)+\lambda_{1}^{(c)}\left(\theta_{c}\right) \delta^{1 / 2}+\ldots
\end{aligned}
$$




\begin{tabular}{|l|lll|llll|}
\hline & \multicolumn{3}{|c|}{ Equation (5.38a) } & \multicolumn{4}{c|}{ Equation (5.38b) } \\
\cline { 2 - 8 } & $O\left(\lambda^{2}\right)$ & $O(\lambda)$ & $O(1)$ & $O\left(\lambda^{2}\right)$ & $O(\lambda)$ & $\begin{array}{c}\sin ^{2} \text { tan } \\
\text { term }\end{array}$ & $\begin{array}{c}\text { sin cos } \\
\text { term }\end{array}$ \\
\hline \hline Case (a) & $\underline{\delta}$ & $\delta^{3 / 2}$ & $\underline{\delta}$ & $\delta^{3 / 2}$ & $\underline{\delta}$ & $\delta^{5 / 2}$ & $\underline{\delta}$ \\
Case (b) & $\underline{1}^{1 / 2}$ & $\delta$ & $\underline{\delta}^{1 / 2}$ & $\underline{\delta}^{1 / 2}$ & $\underline{\delta}^{1 / 2}$ & $\delta^{3 / 2}$ & $\underline{\delta}^{1 / 2}$ \\
Case (c) & $\underline{\delta}^{-1}$ & $\delta^{1 / 2}$ & $\underline{\delta}^{-1}$ & $\underline{\delta}^{-1 / 2}$ & 1 & $\delta^{1 / 2}$ & 1 \\
\hline
\end{tabular}

Table 2: Orders at which the various terms in (5.38) contribute

If these series are substituted into (5.38), the various terms in the equations contribute at the orders shown in Table 2 . Terms that participate in dominant balance are underlined.

Below, we present a few of the low-order terms that result from this calculation. Since our interest in the series is to understand the behavior of $\operatorname{Re}(\lambda)$, in presenting results we ignore higher-order contributions to $\operatorname{Im}(\lambda)$. For consistency, in Case (a) we write $\theta_{a}$ for $\theta$.

$$
\begin{aligned}
\text { Case (a): } \operatorname{Im}(\lambda) & \sim \pm \sqrt{A E} \theta_{a} \delta^{1 / 2}+O\left(\delta^{3 / 2}\right) \\
\operatorname{Re}(\lambda) & \sim\left(\frac{4 C}{D}-\frac{B E}{2}\right) \delta+\left(-\frac{4 A C}{D^{3} E} \theta_{a}^{2}+\frac{16 C^{2}(C-3 A)}{3 A D^{3} E}+\frac{8 B C^{2}}{A D^{2}} \frac{1}{\theta_{a}^{2}}\right) \delta^{2}+O\left(\delta^{3}\right) \\
\text { Case (b):Im }(\lambda) & \sim \pm \sqrt{A E} \theta_{b}+O(\delta) \\
\operatorname{Re}(\lambda) & \sim\left(\frac{4 C D E}{A \theta_{b}^{2}+D^{2} E}-\frac{B E}{2}\right) \delta+O\left(\delta^{2}\right) \\
\text { Case (c): } \operatorname{Im}(\lambda) & \sim \pm 2 \sqrt{A E} \sin \left(\theta_{c} / 2\right) \delta^{-1 / 2}+O\left(\delta^{3 / 2}\right) \\
\operatorname{Re}(\lambda) & \sim-\frac{B E}{2} \delta+\frac{C D E}{A} \cot ^{2}\left(\theta_{c} / 2\right) \delta^{2}+O\left(\delta^{3}\right)
\end{aligned}
$$

These results provide an analytical explanation of much of the behavior of the eigenvalues illustrated in Figure 6; specifically,

- In the perturbation-theory calculation of Section 4.1, we found all the Type II eigenvalues lying along a line $\{\operatorname{Re}(\lambda)=(4 C / D-B E / 2) \delta\}$. The series in Case (a) shows how, to higher order, the eigenvalues recede from this line as $k$ increases.

- In Case (b), the functions of $\theta_{b}$ in $\operatorname{Im}(\lambda)$ and $\operatorname{Re}(\lambda)$ generate the curve along which the eigenvalues lie.

- For $k$ large, the Type II eigenvalues approach the line $\{\operatorname{Re}(\lambda)=(-B E / 2) \delta\}$ along which the Type I eigenvalues lie. The series in Case (c) quantifies this convergence.

Remarks (on the calculation of these series): (i) In finding the coefficients, it is not necessary to solve simultaneous linear equations; rather the coefficients can 
be determined by "back substitution". One can determine the leading order term in the series for $\lambda$, say $\lambda_{\text {lead }}$, from (5.38a) without knowing $\kappa_{1}$; given $\lambda_{\text {lead }}$, one can compute $\kappa_{1}$ from (5.38b); then one may compute $\lambda_{\text {lead }+1}$ from(5.38a) without knowing $\kappa_{2}$; etc. (ii) In Case (c), several of the low-order coefficients vanish: $\kappa_{1}^{(c)}=$ $\lambda_{0}^{(c)}=\lambda_{1}^{(c)}=0$. A hint that $\kappa_{1}^{(c)}$ vanishes may be seen in Table 2 since only one term from $(5.38 \mathrm{~b})$ contributes to dominant balance. The vanishing of the $\lambda^{(c)}$ coefficients can be seen only by doing the calculation.

With the $O\left(\delta^{2}\right)$-correction terms in (5.41), we may demonstrate matching to leading order between the various cases. First consider Cases (a) and (b). Letting $\theta_{a} \rightarrow \infty$ in (5.41a), we obtain

$$
\operatorname{Re}(\lambda) \sim\left(\frac{4 C}{D}-\frac{B E}{2}\right) \delta-\left(\frac{4 A C}{D^{3} E} \theta_{a}^{2}\right) \delta^{2} ;
$$

and substituting $\theta_{a}=\delta^{-1 / 2} \theta_{b}$, this becomes

$$
\operatorname{Re}(\lambda) \sim\left(\frac{4 C}{D}-\frac{B E}{2}-\frac{4 A C}{D^{3} E} \theta_{b}^{2}\right) \delta,
$$

which reproduces the limiting behavior of $(5.41 \mathrm{~b})$ as $\theta_{b} \rightarrow 0$. For Cases (b) and (c), letting $\theta_{c} \rightarrow 0$ in $(5.41 \mathrm{c})$, we obtain

$$
\operatorname{Re}(\lambda) \sim-\frac{B E}{2} \delta+\frac{4 C D E}{A \theta_{c}^{2}} \delta^{2} ;
$$

and substituting $\theta_{c}=\delta^{1 / 2} \theta_{b}$, this becomes

$$
\operatorname{Re}(\lambda) \sim\left(-\frac{B E}{2}+\frac{4 C D E}{A \theta_{b}^{2}}\right) \delta,
$$

which reproduces the limiting behavior of (5.41b) as $\theta_{b} \rightarrow \infty$.

\section{Numerical simulations}

In this section we describe computational results from simulations of one-dimensional solutions of the elasto-plastic model $(2.2,2.3)$. We use these simulations: (i) to numerically compute the eigenvalues for the linear stability of the single-shear-band equilibrium solution, (ii) to verify the prediction of when this equilibrium solution loses stability, (iii) to examine the dynamics of the system when this equilibrium solution is unstable, and (iv) to exhibit the coarsening dynamics in the transient converging to this equilibrium solution when it is stable.

\subsection{Numerical formulation of the one-dimensional elasto-plastic model}

Our numerical study is based on a generalization of (2.24) that describes elastoplastic evolution. In the analysis above, we restricted our attention to solutions 
of $(2.2,2.3)$ in which the deformation was plastic everywhere. In the numerical simulations, we relax this assumption, considering solutions that may be either elastic or plastic. Substituting the ansatz for one-dimensional solutions (2.15) into the nondimensionalized versions of $(2.2,2.3)$, we obtain the governing equations,

$$
\begin{aligned}
\frac{\partial w}{\partial t} & =\frac{\partial p}{\partial x} \\
\mathcal{E}^{-1} \frac{\partial}{\partial t}\left(\begin{array}{c}
p \\
q
\end{array}\right) & =\left(\begin{array}{c}
a+\partial_{x} w \\
b
\end{array}\right)-\mathcal{P}\left(p, q, \partial_{x} w\right)
\end{aligned}
$$

where

$$
\mathcal{P}\left(p, q, \partial_{x} w\right)= \begin{cases}\frac{p\left[a+\partial_{x} w\right]+q b}{\cos \alpha} \mathbf{R}_{\alpha}^{\top}(p, q)^{\top} & \text { if } p^{2}+q^{2} \geq 1 \text { and } p\left[a+\partial_{x} w\right]+q b \geq 0 \\ 0 & \text { otherwise. }\end{cases}
$$

To facilitate numerical simulations of model (6.1), we need to regularize the plastic-strain term (6.1c). We take $\epsilon>0$ to be a regularization parameter and define the indicator functions,

$$
\chi_{\epsilon}(z)=\frac{1}{2}\left(1+\tanh \left(\left[z^{2}-1\right] / \epsilon\right)\right), \quad \psi_{\epsilon}(z)=\frac{1}{2}(z+\epsilon \ln (2 \cosh [z / \epsilon])) .
$$

For $\epsilon \rightarrow 0$ these functions approach the distributions for the yield indicator,

$$
\lim _{\epsilon \rightarrow 0} \chi_{\epsilon}(z)= \begin{cases}0 & |z|<1 \\ 1 & |z| \geq 1\end{cases}
$$

and the loading indicator,

$$
\lim _{\epsilon \rightarrow 0} \psi_{\epsilon}(z)=\max (z, 0),
$$

respectively. Consequently, the regularized form (6.1) can be written as

$$
\begin{aligned}
\frac{\partial w}{\partial t} & =\frac{\partial p}{\partial x} \\
\mathcal{E}^{-1} \frac{\partial}{\partial t}\left(\begin{array}{c}
p \\
q
\end{array}\right) & =\left(\begin{array}{c}
a+\partial_{x} w \\
b
\end{array}\right)-\frac{\chi_{\epsilon}\left(\sqrt{p^{2}+q^{2}}\right)}{\cos \alpha} \psi_{\epsilon}\left(p\left[a+\partial_{x} w\right]+q b\right) \mathbf{R}_{\alpha}^{\top}\left(\begin{array}{c}
p \\
q
\end{array}\right)(6
\end{aligned}
$$

We discretize (6.5) in space using the approach described in Section 2.4. The result is a system of $3 N$ ordinary differential equations that approximate the solution of the continuous system (6.5) through the method of lines. We write this system in standard form as

$$
\frac{d \mathbf{U}}{d t}=\mathbf{F}(\mathbf{U})
$$

with $\mathbf{U}, \mathbf{F} \in \mathbb{R}^{3 N}$,

$$
\mathbf{U}=\left(\mathbf{u}_{0}, \mathbf{u}_{1}, \cdots, \mathbf{u}_{N-1}\right)^{\top}, \quad \mathbf{F}(\mathbf{U})=\left(\mathbf{f}_{0}, \mathbf{f}_{1}, \cdots, \mathbf{f}_{N-1}\right)^{\top} .
$$


The components of these vectors are themselves vectors in $\mathbb{R}^{3}$. The local solution in each grid cell is $\mathbf{u}_{n}=\left(w_{n}, p_{n}, q_{n}\right)^{\top} \in \mathbb{R}^{3}$. The right-hand sides of (6.5) in each grid cell, $\mathbf{f}_{n} \in \mathbb{R}^{3}$, are given in terms of the values of $\mathbf{u}_{n}, \mathbf{u}_{n+1}$, and $\mathbf{u}_{n-1}$ by

$$
\mathbf{f}_{n}=\mathcal{F}\left(\frac{w_{n+1}-w_{n}}{\delta}, \frac{p_{n}-p_{n-1}}{\delta}, p_{n}, q_{n}\right),
$$

where the function $\mathcal{F}: \mathbb{R}^{4} \rightarrow \mathbb{R}^{3}$ is defined by

$$
\mathcal{F}\left(w_{x}, p_{x}, p, q\right) \equiv\left(\mathcal{E}\left[\left(\begin{array}{c}
a+w_{x} \\
b
\end{array}\right)-\frac{\chi_{\epsilon}\left(\sqrt{p^{2}+q^{2}}\right)}{\cos \alpha} \psi_{\epsilon}\left(p\left[a+w_{x}\right]+q b\right) \mathcal{R}_{\alpha}^{\top}\left(\begin{array}{c}
p \\
q
\end{array}\right)\right]\right) .
$$

Periodic boundary conditions are imposed by specifying that $w_{N}(t)=w_{0}(t)$ in $\mathbf{f}_{N-1}$ and that $p_{-1}(t)=p_{N-1}(t)$ in $\mathbf{f}_{0}$. This choice of relations is slightly different than (2.25), but it yields an equivalent system of equations and is more convenient for putting (6.5) into standard form.

With the problem written in standard form, it is straightforward to use standard numerical methods for initial value problems to solve the system of ordinary differential equations. Explicit time-stepping schemes, including forward Euler and Runge-Kutta methods, can be applied directly to (6.6). Solving (6.6) using implicit methods, such as a backward Euler scheme, involves calculating the Jacobian matrix or functional derivative of the system. For $\epsilon=0$, because of the non-smooth distributions $(6.3,6.4)$, this derivative does not exist, but this difficulty is not an issue for the regularized forms (6.2). We found that to ensure stability, a very small time-step was necessary: $\Delta t$ had to be much smaller than $\delta / \sqrt{\mathcal{E}}$, the upper bound on $\Delta t$ expected from the CFL condition for elastic waves in (6.1) with $\mathcal{P}=0$. This suggests that the influence of the regularized plastic yield condition is to make (6.6) a very stiff ODE system. We validate this suggestion in the next section with a direct calculation of the spectrum for the linearized system for (6.6).

\subsection{Linear stability of the single shear band equilibrium}

Calculating the Jacobian, $\mathbf{J}=\partial \mathbf{F} / \partial \mathbf{U}$, of (6.6) evaluated at the single-shear-band equilibrium yields a numerical representation the matrix for the linearized operator for this system. We can then readily obtain the eigenvalues of the resulting sparse matrix numerically to yield the spectrum of the linear stability problem. For fixed values of $\delta=1 / N$ and $E=\mathcal{E} / \delta$, we numerically examined the spectrum in the limit $\epsilon \rightarrow 0$. The resulting set of $3 N$ eigenvalues obtained from the Jacobian matrix may be characterized as follows:

1. $2 N$ of the eigenvalues converge to finite limits as $\epsilon \rightarrow 0$.

2. $N$ of the eigenvalues diverge like $\operatorname{Re}(\lambda)=O\left(\epsilon^{-1 / 5}\right) \rightarrow-\infty$ as $\epsilon \rightarrow 0$. 


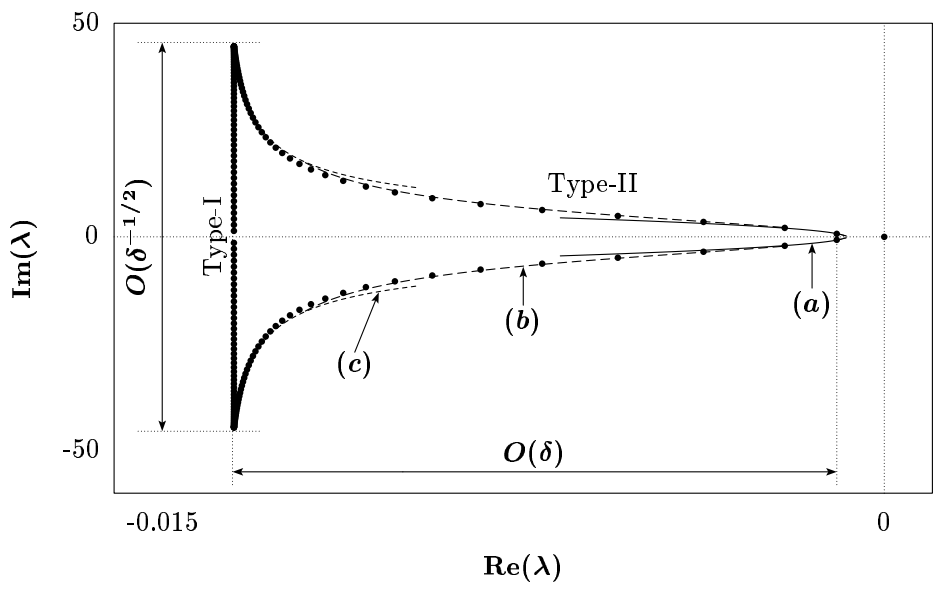

Figure 5: Numerical calculation of the spectrum of $(6.6-6.9)$ for $N=100, \mathcal{E}=5 \delta$, $\alpha=\pi / 4$ (dots), compared with asymptotic results given by equations (5.41abc) (curves).

In the limit $\epsilon \rightarrow 0$, the set of the $2 N$ finite eigenvalues coincide with the eigenvalues analyzed in Section 4. This is illustrated in Figure 5, which shows the computed eigenvalues for $N=100$ and $\epsilon=10^{-4}$ as well as the graphs of the asymptotic formulas $(5.41 \mathrm{abc})$. Note the great disparity between the horizontal and vertical scales in the figure. The large-negative Type II eigenvalue, $\lambda \approx-D E$, lies far outside the narrow domain shown in the figure.

The second set of $N$ eigenvalues diverge at a rate controlled by the regularization for $\epsilon \rightarrow 0$ in (6.5). As noted at the end of Section 2.1, when the yield condition is a sharp constraint (i.e. for $\epsilon=0$ ), infinitesimal elastic perturbations decay to zero in a small finite time. Within the framework of linear stability analysis, such perturbations vanish faster than would be expected with any finite eigenvalue, hence heuristically, we can describe these modes of evolution as having infinite eigenvalues, $\lambda=-\infty$. For $\epsilon>0$, when the yield condition is smoothed out by (6.2), the evolution of these perturbations is modified to a rapid exponential decay at the rate given above. Incidentally, there are only two distinct eigenvalues in this second group, a simple real eigenvalue $\lambda=O\left(\delta^{1 / 5} \epsilon^{-1 / 5}\right)$, and a second real eigenvalue $\lambda=O\left(\delta \epsilon^{-1 / 5}\right)$ that has (algebraic and geometric) multiplicity $N-1$.

The singular behavior of these eigenvalues as $\epsilon \rightarrow 0$ is clarified by a differentialalgebraic-equations viewpoint. In Sections 3 and 4 we analyzed stability of equilibrium solutions of (2.24) by using the linearized yield condition to eliminate $N$ of the $3 N$ unknowns in this equation, leading to an (ordinary) eigenvalue problem $\mathbf{A x}=\lambda \mathbf{x}$. Suppose instead, as in the Remark at the end of Section 2.4, we retained all $3 N$ unknowns in a differential-algebraic system of equations consisting of the same $2 N$ differential equations as before plus $N$ algebraic equations coming from 

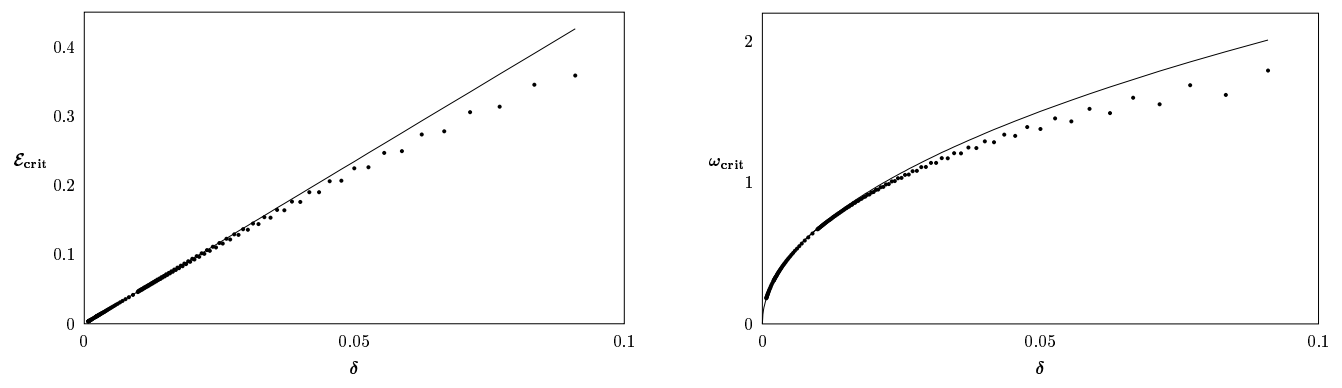

Figure 6: Numerical calculation of the stability criterion, $\mathcal{E}_{\text {crit }}$, and the oscillation frequency at marginal stability $\omega_{\text {crit }}$ (dots) compared with the asymptotic results (6.10) and (6.11) (curves) respectively, as functions of $\delta=1 / N$ for $N=10,11, \cdots$.

the yield condition. In this case, the stability analysis would lead to a generalized eigenvalue problem $\tilde{\mathbf{A}} \mathbf{x}=\lambda \mathbf{B} \mathbf{x}$. Since $\mathbf{B}$ has rank $2 N$, the additional $N$ generalized eigenvalues are all infinite (see [15]).

\subsection{The Hopf bifurcation}

We use the computation of the spectrum of the linear operator to verify the dependence of the stability criterion for the equilibrium solution on $\delta$, as given in (4.1). In Figure 6(left), the dots indicate the numerically computed critical values of $\mathcal{E}$ for $\delta=1 / N$ as $N \rightarrow \infty$. The solid line is the graph of leading order term from

$$
\mathcal{E}_{\text {crit }}(\delta)=\left(\frac{2 \sin ^{2}(2 \alpha)}{\sin (2 \alpha-\mu)}\right)^{2} \delta+O\left(\delta^{2}\right),
$$

as in (4.1). As the figure shows, the numerical results are well described by the asymptotics. Regarding assumption (5.3), note from these plots that for small values of $N$ the differences between $N$ even and $N$ odd are observable, but they become negligible in the continuum limit, $N \rightarrow \infty$.

When $\mathcal{E}=\mathcal{E}_{\text {crit }}(\delta)$, the imaginary part of the marginally stable eigenvalue computed from (5.41a) with $\theta_{a}=\pi$ is

$$
\omega_{\text {crit }}(\delta)=\frac{2 \sqrt{2} \sin ^{2}(2 \alpha) \sin \alpha}{\sin (2 \alpha-\mu)} \sqrt{\delta}+O\left(\delta^{3 / 2}\right) .
$$

The leading order term in this function and the computed eigenvalues are graphed in Figure 6(right); again the agreement is good. Since $\omega_{\text {crit }} \neq 0$, as $\mathcal{E}$ crosses $\mathcal{E}_{\text {crit }}$ a Hopf bifurcation occurs.

\subsection{Simulations of dynamics following the loss of stability}

Figure 7 is a numerically calculated bifurcation diagram showing branches of stable solutions of $(6.5)$ as a function of the control parameter $\mathcal{E}$. The branches are 


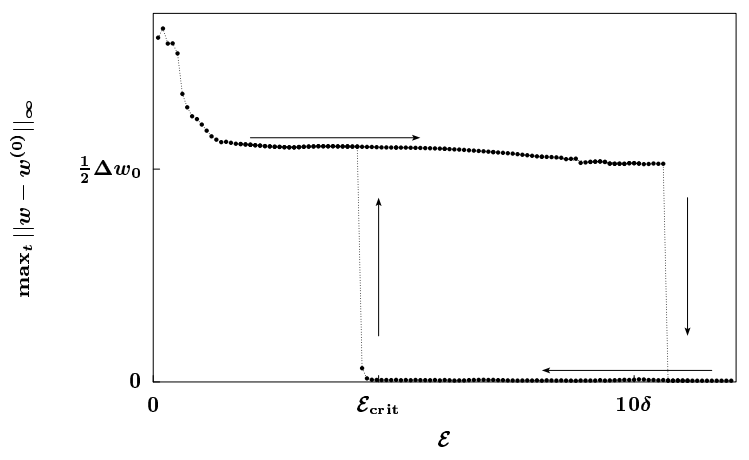

Figure 7: A numerically calculated bifurcation diagram - branches of stable solutions of the discrete elasto-plastic model. The maximum deviation of the velocity profile from the equilibrium shear band, $\max _{t}\left\|w(t)-w^{(0)}\right\|_{\infty}$, is plotted as a function of $\mathcal{E}$ for $N=100$.

plotted in terms of their maximum deviation from the velocity profile of the singleshear-band equilibrium; hence the equilibrium solution corresponds to the trivial branch in the the figure. Verifying the eigenvalue computation above, the equilibrium solutions indeed lose stability for $\mathcal{E} \leq \mathcal{E}_{\text {crit }}(\delta)$. This appears to happen as a subcritical Hopf bifurcation, as the solutions abruptly jump to a branch of stable finite-amplitude periodic solutions. The structure of these periodic solutions includes propagating elastic waves that interact with and reflect off of the underlying shear band, see Figure 8b, where the shear band is placed in the middle of the domain for convenience. The amplitude of the elastic waves is comparable with that of the steady shear band, $\Delta w_{0}$ given by (3.6); indeed, during part of the period, the elastic waves nearly double the amplitude of the jump in the velocity, while at other times, they nearly eliminate the jump. Note that in these solutions, the stress at the shear band always remains at yield, $|\boldsymbol{\tau}|=1$, with value

$$
\boldsymbol{\tau} \approx \boldsymbol{\tau}_{0}^{(0)}=(\cos \phi, \sin \phi)^{\top},
$$

but the stress in the surrounding regions changes in response to the elastic waves. The branch of periodic solutions extends to values of $\mathcal{E}$ larger than $\mathcal{E}_{\text {crit }}$ but eventually does lose stability, decaying to the equilibrium solution (see Fig 7).

Incidentally, the behavior of the periodic solution in the limit $\mathcal{E} \rightarrow 0$ was not resolved by these simulations. We plan to explore this limit in a future publication.

\subsection{Coarsening dynamics}

In [6] we studied the dynamics of the PDE which is the formal limit of $(2.2,2.3)$ as $\mathcal{E} \rightarrow \infty$. Starting from a slight perturbation of the uniform solution (2.14), for which 

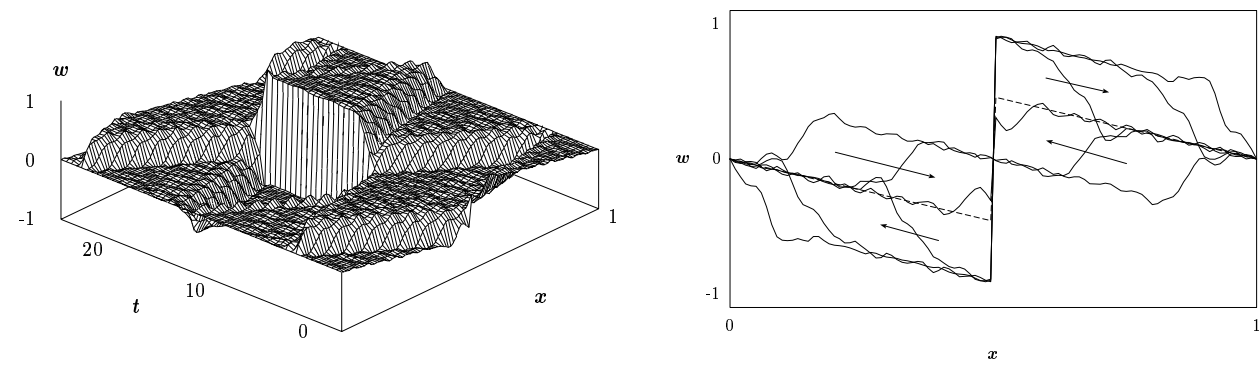

Figure 8: Dynamics of the stable periodic solutions: (left) the velocity plotted as a function of space and time over one period, (right) the periodic velocity profile (solid) compared with the equilibrium shear band (dashed); directions of propagation of the elastic waves are shown.

the PDE is ill-posed, grid-scale oscillations quickly formed in the discrete model. These oscillations can be associated with localized shear bands. It was shown in [6] that solutions with multiple shear bands are unstable; hence a coarsening process ensues, in which some shear bands grow in amplitude while others collapse. This process successively reduces the number of shear bands, until ultimately the solution converges to a single stable shear band. It was found that, in a time interval $O\left(N^{-2}\right)<t<O(N)$, the number of shear bands in the system at a given time, $K(t)$, obeys the self-similar scaling law

$$
\frac{K(t)}{N} \sim C\left(N^{2} t\right)^{-1 / 3} .
$$

We look for similar behavior in (2.24) when the one-shear-band equilibrium solution is stable (i.e., for $\mathcal{E}$ above $\mathcal{E}_{\text {crit }}(\delta)$ ). For this purpose we define a shear band in the transient as a grid point where a stress defect occurs: i.e., a grid point $n$ where $q_{n}>0$, rather than $q_{n}<0$, which holds at all points away from the shear band, see (3.8b). Let $K(t)$ be the number of grid points where $q_{n}>0$ at time $t$. Figure 9 shows the evolution of $K(t)$ for simulations of (6.6) at several different values of $\mathcal{E}$ starting from small perturbations of the uniform equilibrium (2.14). For small to moderate values of $\mathcal{E}$, there is a noticeable delay before the onset of coarsening; for a substantial time $K(t)$ remains close to $N$ before falling off rapidly. During these transient stages of the evolution, the solution in much of the domain has stresses below the yield surface. For sufficiently long times, the stresses approach the yield surface almost everywhere. This convergence to plastic evolution is accompanied by a convergence to the scaling law (6.13) for $K(t)$. For larger values of $\mathcal{E}$, the convergence to the dynamics for the plastic limit $(\mathcal{E}=\infty)$ occurs more rapidly. 


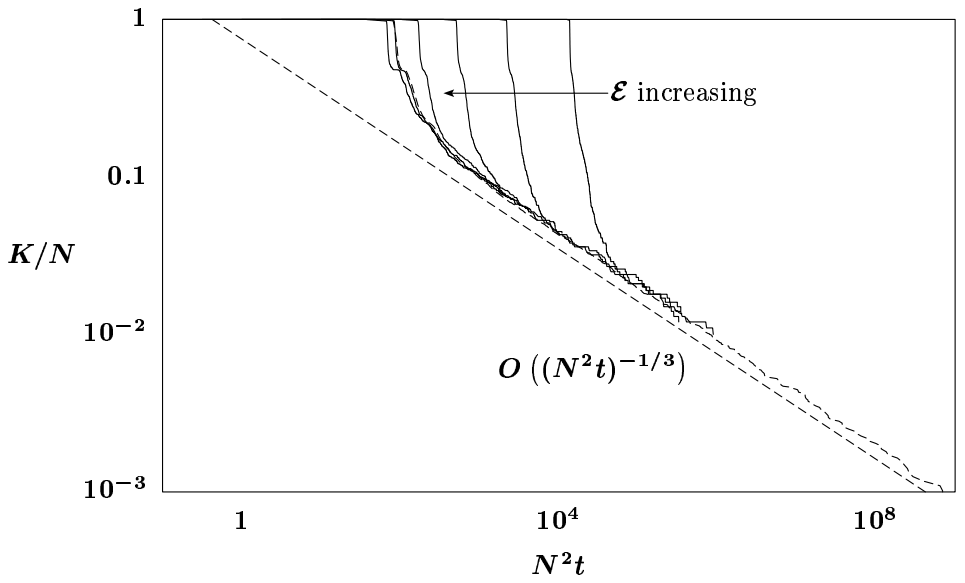

Figure 9: Convergence of the coarsening dynamics of the elasto-plastic system to (6.13) as $\mathcal{E} \rightarrow \infty$. Simulations with $N=1000$ for $\mathcal{E}=10^{k}$ for $k=2,3, \cdots, 7$ (solid curves) converge to results from a simulation of the parabolic PDE for $\mathcal{E}=\infty$ (dashed curve). A dashed line of slope $-1 / 3$ is also shown for comparison.

\section{Discussion}

In this paper, we have discretized an ill-posed PDE model for granular flow, and examined the stability of shear band equilibrium solutions in the discretized equations. We found that stability is lost as the elastic modulus decreases below a critical value $\mathcal{E}_{\text {crit }}(\delta)$, where $\delta>0$ is the lengthscale for the grid discretization. The parameter $\delta$ can be regarded as representing the size of an individual grain, and the finite-difference discretization roughly reflects the discreteness of the granular material. It could perhaps be argued that the discrete model represents a particular law of nearest-neighbor interactions between grains in a very dense flow. It is not our intention to attempt to justify the discretized model from a micromechanical basis. We use the model to illustrate the influence of discreteness (in whatever micro-mechanical form it might take) as a modification of the underlying continuum mechanics model of the flow.

As stated in the introduction, the motivation for this paper and its predecessor [6] is to make sense of ill-posed dynamic equations that are continuum models for the intrinsically discrete medium of granular materials. These ideas may be useful in other contexts, such as fracture [16-18] and strain softening in metals [19-22], where discreteness may play a crucial role in regularizing continuum equations that exhibit solutions with singular behavior.

Towards the end of this paper we discuss preliminary numerical results concerning the periodic solutions that appear as a result of the loss of stability of equilibrium shear band solutions. It seems that as the shear modulus $\mathcal{E}$ is decreased further, 
there are period doubling bifurcations. One might speculate that the true dynamics at realistic (i.e., small) values of $\mathcal{E}$ are chaotic (cf. also [23]), behavior that may be related to the stress fluctuations observed in experiments with granular material undergoing slow shearing $[10,24]$.

\section{References}

[1] R. Hill. Acceleration waves in solids. J. Mech. Phys. Solids, 10:1-16, 1962.

[2] J. Mandel. Condition de stabilité et postulat de Drucker. In J. Kravtchenko and P. Sirieys, editors, Rheology and Soil Mechanics (IUTAM Symposium at Grenoble), pages 58-68. Springer, Berlin, 1966.

[3] J. Rice. The localization of plastic deformation. In W. Koiter, editor, Proc. 14th IUTAM Congress, pages 207-220. Delft, 1976.

[4] J. Rice and J. Rudnicki. Conditions for the localization of deformation in pressure-sensitive dilatant materials. J. Mech. Phy. Solids, 23:371-394, 1975.

[5] D. G. Schaeffer. Instability in the evolution equations describing incompressible granular flow. J. Differential Equations, 66(1):19-50, 1987.

[6] T. P. Witelski, D. G. Schaeffer, and M. Shearer. A discrete model for an ill-posed nonlinear parabolic PDE. Physica D, 160:189-221, 2001.

[7] D. G. Schaeffer. A mathematical model for localization in granular flow. Proc. Roy. Soc. London Ser. A, 436(1897):217-250, 1992.

[8] I. Vardoulakis and J. Sulem. Bifurcation analysis in geomechanics. Blackie Academic and Professional/Chapman and Hall, London, 1995.

[9] G. Capriz, V. N. Ghionna, and P. Giovine. Modeling and mechanics of granular and porous materials. Birkhauser, Boston, 2002.

[10] R.P. Behringer, J. Geng, D. Howell, E. Longhi, G. Reydellet, L. Vanel, and E. Clement. Fluctuations in granular materials. In Y. Kishino, editor, Powders and Grains 2001, pages 347-354. A. A. Balkema Publishers, Rotterdam, the Netherlands, 2001.

[11] D. G. Schaeffer and M. Shearer. Scale-invariant initial value problems in onedimensional dynamic elastoplasticity, with consequences for multidimensional nonassociative plasticity. European J. Appl. Math., 3(3):225-254, 1992.

[12] I. Vardoulakis and B. Graf. Calibration of constitutitive models for granularmaterials using data from biaxial experiments. Geotechnique, 35(3):299-317, 1985. 
[13] I. Stakgold. Green's functions and boundary value problems. John Wiley \& Sons, New York, 1979.

[14] B. L. van der Waerden. Modern Algebra. Vol. I. Frederick Ungar Publishing Co., New York, N. Y., 1949.

[15] K. E. Brenan, S. L. Campbell, and L. R. Petzold. Numerical solution of initialvalue problems in differential-algebraic equations. Society for Industrial and Applied Mathematics (SIAM), Philadelphia, PA, 1996.

[16] G. Puglisi and L. Truskinovsky. Mechanics of a discrete chain with bi-stable elements. J. Mech. Phys. Solids, 48:1-27, 2000.

[17] R. C. Rogers and L. Truskinovsky. Discretization and hysteresis. Physica B, 233:370-375, 1997.

[18] L. Truskinovsky. Fracture as a phase transition. In R.C. Batra and M.F. Beatty, editors, Contemporary research in the mechanics andmathematics of materials. A collection of papers dedicated to the 70th birthday of J.L. Ericksen, pages 322-332. CIMNE, Barcelona, 1996.

[19] A. Bayliss, T. Belytschko, M. Kulkarni, and D. A. Lott-Crumpler. On the dynamics and the role of imperfections for localization in thermo-viscoplastic materials. Modelling Simul. Mater. Sci. Eng., 2:941-964, 1994.

[20] J. A. DiLellio and W. E. Olmstead. Shear band formation due to a thermal flux inhomogeneity. SIAM J. Appl. Math., 57(4):959-971, 1997.

[21] W. E. Olmstead, S. Nemat-Nasser, and L. Ni. Shear bands as surfaces of discontinuity. J. Mech. Phys. Solids, 42:697-709, 1994.

[22] J. W. Walter. Numerical experiments on adiabatic shear band formation in one dimension. Int. J. Plasticity, 8:657-693, 1992.

[23] J. M. Ottino and D. V. Khakhar. Open problems in active chaotic flows: competition between chaos and order in granular materials. Chaos, 12(2):400407, 2002.

[24] D. Howell, R.P. Behringer, and C. Veje. Stress fluctuations in a 2d granular couette experiment: a continuous transition. Phys. Rev. Lett., 82:5241-5244, 1999. 